\title{
A Priori Field Study of the Subgrid-Scale Heat Fluxes and Dissipation in the Atmospheric Surface Layer
}

\author{
FERnANdo Porté-Agel \\ Department of Civil Engineering and St. Anthony Falls Laboratory, University of Minnesota, Minneapolis, Minnesota
}

\author{
Marc B. PARLANGe
}

Department of Geography and Environmental Engineering, and Center for Environmental and Applied Fluid Mechanics, The Johns Hopkins University, Baltimore, Maryland

Charles Meneveau

Department of Mechanical Engineering, Center for Environmental and Applied Fluid Mechanics, The Johns Hopkins University, Baltimore, Maryland

WILLIAM E. EICHINGER

Department of Civil and Environmental Engineering, Iowa Institute for Hydraulic Research, University of Iowa, Iowa City, Iowa

(Manuscript received 20 January 2000, in final form 27 September 2000)

\begin{abstract}
Field measurements are carried out to study statistical properties of the subgrid-scale (SGS) heat fluxes and SGS dissipation of temperature variance in the atmospheric surface layer, and to evaluate the ability of several SGS models to reproduce these properties. The models considered are the traditional eddy-diffusion model, the nonlinear (gradient) model, and a mixed model that is a linear combination of the other two. High-resolution wind velocity and temperature fields are obtained from arrays of 3D sonic anemometers placed in the surface layer. The basic setup consists of two horizontal parallel arrays (seven sensors in the lower array and five sensors in the upper array) at different heights (2.4 and $2.9 \mathrm{~m}$, respectively). Data from this setup are used to compute the SGS heat flux and dissipation of temperature variance by means of 2D filtering in horizontal planes, invoking Taylor's hypothesis. Model coefficients are measured from the data by requiring the real and modeled timeaveraged dissipation rates to match. Various other experimental setups that differ mainly in the separation between the sensors are utilized to show that filter size has a considerable effect on the various model coefficients near the ground. For the basic setup, conditional averaging is used to study the relation between large-scale coherent structures (sweeps and ejections) and the SGS quantities. It is found that under unstable conditions, negative SGS dissipation, indicative of backscatter of temperature variance from the subgrid scales to the resolved field, is most important during the onset of ejections transporting relatively warm air upward. Large positive SGS dissipation of temperature variance is associated with the end of ejections (and/or the onset of sweeps) characterized by strong drops in temperature and vertical velocity under unstable conditions. These results are also supported by conditionally sampled 2D (streamwise and vertical) velocity and temperature distributions, obtained using an additional setup consisting of the 12 anemometers placed in a vertical array. The nonlinear and mixed model reproduce the observations better than the eddy-diffusion model.
\end{abstract}

\section{Introduction}

Due to recent developments in computer resources, numerical simulations, particularly large-eddy simulations (LESs), have become a powerful tool used to study the unsteady 3D transport of momentum and scalar quantities in turbulent flows. Applied to the atmospheric

Corresponding author address: Dr. Marc B. Parlange, Department of Geography and Environmental Engineering, The Johns Hopkins University, 313 Ames Hall, Baltimore, MD 21218-2686.

E-mail: mbparlange@jhu.edu boundary layer (ABL), simulations can help disentangle the role that different variables such as surface heterogeneity and atmospheric stability play on the removal and mixing of heat, water vapor, and pollutants (Moeng 1984; Nieuwstadt et al. 1991; Shaw and Schumann 1992; Andrén et al. 1994; Sullivan et al. 1994; Parlange et al. 1995; Sorbjan 1996; Dwyer et al. 1997; Avissar and Schmidt 1998; Albertson and Parlange 1999a,b).

In 3D simulations of high-Reynolds-number turbulent flows (e.g., the $\mathrm{ABL}$ ), computational limitations impose the choice of a grid or mesh size substantially larger 
than the smallest scale of motion (Kolmogorov scale $\eta$ $\sim 1 \mathrm{~mm}$ in the ABL). Large-eddy simulation (LES) deals with this limitation by resolving the transport equations for all scales of motion larger than the grid size $\Delta_{\text {LES }}$, while the contribution of the subgrid scales (smaller than $\Delta_{\text {LES }}$ ) on the resolved field is parameterized using a subgrid-scale (SGS) model. Thus, the ability of LES to provide realistic turbulent fields hinges on the realism of the SGS model.

The equations solved during LES for the large scales of motion differ from the original Navier-Stokes momentum and scalar transport equations. The LES equations are obtained by applying a spatial filter of characteristic width $\Delta$ ( $\left.\Delta \geq \Delta_{\text {LES }}\right)$ to the original equations. This yields a set of equations for the evolution of the filtered (resolved) quantities, amenable to numerical solution on a grid with mesh size $\Delta_{\text {LES }}$. In the case of a scalar quantity $\theta$ (e.g., temperature), the effect of the nonresolved (subgrid) scales appears in the governing equation for $\tilde{\theta}$-where the tilde $(\sim)$ denotes the filtering operation at scale $\Delta$-as the divergence of the so-called SGS scalar flux $\left(q_{i}\right)$. In the $i$ direction, the SGS scalar flux is defined as

$$
q_{i}=\widetilde{u_{i} \theta}-\tilde{u_{i}} \tilde{\theta}
$$

where $u_{i}$ is the velocity component in the $i$ direction. This term-particularly the filtered product $\widetilde{u_{i}} \theta$-is unknown and therefore it must be parameterized (using a SGS model) as a function of the resolved field.

The subgrid scales have a very important effect on the level of fluctuations of the resolved turbulent field. A measure of this variability in the resolved scalar field is the filtered scalar variance $\sigma_{\theta}^{2}=\left\langle 1 / 2 \tilde{\theta}^{\prime 2}\right\rangle=1 / 2\langle(\tilde{\theta}-$ $\left.\langle\tilde{\theta}\rangle)^{2}\right\rangle$, where \langle\rangle denotes the ensemble average and the prime is the departure from that average $(\tilde{\theta}=\langle\tilde{\theta}\rangle+$ $\left.\tilde{\theta}^{\prime}\right)$. The effect of the subgrid scales on the evolution of $\sigma_{\theta}^{2}$ appears through the so-called SGS dissipation rate (or SGS transfer rate) of resolved scalar variance,

$$
\chi=-q_{j} \frac{\partial \tilde{\theta}^{\prime}}{\partial x_{j}},
$$

where $\chi$ is also equal to the production of unresolved scalar fluctuations, and it has a dominant effect on the evolution of $\sigma_{\theta}^{2}$. On average one expects a net drain of resolved scalar variance into the subgrid scales, that is, $\langle\chi\rangle>0$. For details on the evolution equations for $\tilde{\theta}$ and $\sigma_{\theta}^{2}$ see Porté-Agel et al. (1998). Next, we present a review of the current state of SGS models in LES (with emphasis on the atmospheric boundary layer), and motivate the use of carefully designed field experiments (such as the one presented in this paper) to address open issues in SGS modeling.

\section{a. Subgrid-scale models}

The formulation of the SGS model for the dynamics of the small scales has a particularly important effect in the near-surface region of wall-bounded flows. The reason is that there the LES filter scale is on the order of the distance to the surface, which is also the local integral scale of turbulence. Hence, the idealized assumptions of an inertial range of turbulence do not hold in the near-wall region. Since this region is crucial in determining the overall fluxes, the need to improve the existing models is a key step in making LES a more reliable tool. Next, we present the formulation and main characteristics of some of the most popular SGS models. Many of these are motivated by inertial range dynamics. More details on these models and other recent developments in SGS modeling can be found in Mason (1994), Meneveau and Katz (2000), and Pope (2000).

Eddy-diffusion (eddy-viscosity) models parameterize the SGS fluxes (SGS stresses) as being proportional to the resolved scalar (and velocity) gradients. Particularly, the $i$ component of the SGS heat flux modeled with the eddy-diffusion model is of the form

$$
q_{i}^{\text {ed }}=-\operatorname{Pr}_{T}^{-1} C_{S}^{2} \Delta^{2}|\tilde{S}| \frac{\partial \tilde{\theta}}{\partial x_{i}},
$$

where $|\tilde{S}|=\left(2 \tilde{S}_{i j} \tilde{S}_{i j}\right)^{1 / 2}$ is the resolved strain-rate magnitude, $\tilde{S}_{i j}=1 / 2\left(\partial \tilde{u}_{i} / \partial x_{j}+\partial \tilde{u}_{j} / \partial x_{i}\right)$ is the resolved strainrate tensor, $C_{S}$ is the so-called Smagorinsky coefficient, and $\operatorname{Pr}_{T}$ is the subgrid Prandtl number. When a cutoff filter is used in the inertial subrange of isotropic, homogeneous turbulence, $C_{S}$ is known to have a value of about 0.17 (Lilly 1967). In simulations of high-Reynolds number wall-bounded flows, such as the ABL, the eddyviscosity model appears to have problems in reproducing the correct SGS dissipation rate. First, the model is too dissipative even when empirical matching functions are employed to reduce the value of the coefficient as the ground is approached (Mason 1989; Mason and Thomson 1992; Porté-Agel et al. 2000a). Second, the model is fully dissipative, that is, it does not allow for backscatter of energy or scalar variance from the subgrid scales to the resolved field. Recently, some attempts to overcome these shortcomings have been made, including the use of twopart eddy-viscosity models, in which the mean and fluctuating shear are treated separately (e.g., Sullivan et al. 1994), and also models that include stochastic fluctuations in the SGS stresses to allow for backscatter (Mason and Thomson 1992; Schumann 1995). In both cases the model requires the specification of an additional model coefficient. Another important deficiency of the eddydiffusion model is the low correlation between the measured and modeled SGS stresses (e.g., Clark et al. 1979; Bardina et al. 1980; Liu et al. 1994).

The so-called dynamic model (Germano et al. 1991; Lilly 1967; Ghosal et al. 1995) computes the value of the coefficient in the eddy-viscosity model at every time and position in the flow, based on scale similarity at the smallest resolved scales and assuming scale invariance of the model coefficient. Although successfully implemented in a number of engineering flows (see, e.g., Akselvoll and Moin 1996), the application of the dy- 
namic model to atmospheric flows has not yet become commonplace. Recently, Porté-Agel et al. (2000a) introduced a scale-dependent dynamic model that allows the model coefficient to change with scale. Applications of the scale-dependent model to LES of the ABL show improved dissipative properties (with respect to the standard Smagorinsky and dynamic models), leading to more realistic spectra and mean velocity profiles (PortéAgel et al. 2000a).

Recently, the so-called similarity model and nonlinear (gradient) model have gained popularity. They take advantage of the scale similarity or invariance of the dynamics at different scales in the inertial range of turbulence. The high correlation observed (e.g., Bardina et al. 1980) between the SGS fluxes and the smallest resolved fluxes has led to the formulation of the similarity model, which for the SGS heat flux is (Bardina et al. 1980; Liu et al. 1994)

$$
q_{i}^{\text {sim }}=C_{\text {sim }}\left(\overline{\tilde{u}_{i} \tilde{\theta}}-\overline{\tilde{u}}_{i} \overline{\tilde{\theta}}\right) .
$$

The overbar denotes a second filtering operation, now at some scale $\gamma \Delta$ with $\gamma \geq 1$. The formulation of the similarity model given by Eq. (1.4) involves additional computational expense due to the secondary filtering operations. This can be avoided by expanding Eq. (1.4) in a Taylor series and performing the filtering analytically (Leonard 1974; Clark et al. 1979; Liu et al. 1994). The result is the so-called nonlinear or gradient model,

$$
q_{i}^{\mathrm{nl}}=C_{\mathrm{nl}} \Delta^{2} \frac{\partial \tilde{u}_{i}}{\partial x_{k}} \frac{\partial \tilde{\theta}}{\partial x_{k}}
$$

where $C_{\mathrm{nl}}$ depends on $\gamma$ and filter type. The repeated index $k$ corresponds to the directions involved in the filtering operation (i.e., $k=1,2,3$ for a $3 \mathrm{D}$ filter and $k=1,2$ if a $2 \mathrm{D}$ filter-in the streamwise and spanwise directions-is used). Analysis of DNS data shows elevated correlation between real stresses (measured from the data) and modeled SGS stresses (obtained using the similarity and nonlinear models) (e.g., Bardina et al. 1980). Furthermore, these models are able to reproduce backscatter of energy and scalar variance (see, e.g., Bardina et al. 1980; Liu et al. 1994; Sarghini et al. 1999). However, when implemented in simulations, the similarity model, or the nonlinear model alone, does not dissipate enough energy and typically yields numerically unstable simulations. Faced with this difficulty, Bardina et al. (1980) suggested adding a dissipative eddy-diffusion term. When using the nonlinear model, the resulting mixed model is

$$
q_{i}^{\text {mix }}=-\left[\operatorname{Pr}_{T}^{-1} C_{S}^{2}\right]^{*} \Delta^{2}|\tilde{S}| \frac{\partial \tilde{\theta}}{\partial x_{i}}+\left[C_{\mathrm{nl}}\right]^{*} \Delta^{2} \frac{\partial \tilde{u}_{i}}{\partial x_{k}} \frac{\partial \tilde{\theta}}{\partial x_{k}},
$$

where $\left[\operatorname{Pr}_{T}^{-1} C_{S}^{2}\right]^{*}$ and $\left[C_{\mathrm{n}}\right]^{*}$ are the mixed model coefficients. The mixed model combines the strengths of both the similarity (or nonlinear) model and the eddyviscosity model. Typically the magnitude of the similarity term is significantly higher than that of the Sma- gorinsky term. Hence, the eddy-viscosity term does not degrade the high correlation coefficient (Liu et al. 1995). Although successfully applied in a number of engineering flows (see, e.g., Sarghini et al. 1999; Anderson and Meneveau 1999), to date these mixed models have not been used widely in simulations of the ABL. Kosovic (1997) has used a nonlinear model in LES of the ABL and shows significant improvements in the predicted flow field as compared to the simple eddy-viscosity model.

\section{b. A priori field studies}

One of the major difficulties encountered when attempting to improve SGS model formulations is our limited understanding of the most fundamental characteristics and dynamics of the SGS variables (such as fluxes and dissipation), which need to be reproduced by the SGS model. Moreover, even for a given model formulation, the optimal value of the model coefficient(s) as a function of time and position in the flow is uncertain. Two major complementary approaches have been taken toward the improvement of SGS models for LES, namely a posteriori tests and a priori studies. A posteriori tests evaluate SGS models based on the comparison between the statistical characteristics of the flow obtained from the simulations and those measured in the ABL. A limitation of a posteriori tests is that it is often difficult to disentangle the effect of the SGS model from the other factors such as numerical resolution, discretization methods, parameterization of surface heterogeneity, etc. Hence, they only yield indirect evidence about the true nature of SGS dynamics. A priori studies use well-resolved turbulent fields (from DNS of relatively small Reynolds number flows or from experiments) from which we can learn about the characteristics of the SGS dynamics that need to be captured by the SGS model. This approach was first used with data from DNS of isotropic turbulence (e.g., Clark et al. 1979; Domaradski et al. 1993), other turbulent flows (e.g., Piomelli et al. 1988; Härtel et al. 1994; Piomelli et al. 1996), and also with laboratory measurements (Meneveau 1994; Liu et al. 1994; Meneveau and Katz 1999b; Bastiaans et al. 1998). In high-Reynolds number turbulent boundary layers, in particular in the ABL, there is a growing recognition (see Tong et al. 1998) of the need for field experiments that are designed to provide information about the nature of the SGS variables in these flows.

One particularly important subject is the relation between the SGS variables and large-scale features of the flow such as coherent structures. Strong direct effects of coherent structures in a plane wake (roller vortices in the von Kármán street) upon SGS variables were observed experimentally by O'Neil and Meneveau (1997). In boundary layer flows, coherent structures are associated with the sweep/ejection nature of the flow and their presence in the atmospheric surface layer is best identified through ramps (sawtooth structures) in the measured tem- 
perature signal (e.g., Gao et al. 1989; Shaw et al. 1983, 1989; Gao and Shaw 1992; Raupach et al. 1991; Katul et al. 1997b; Szilagyi et al. 1999). For example, under unstable (convective) conditions, ejection events bring relatively hot air upward and they are associated with increments in the temperature, while sweep events bringing relatively cool air downward are associated with temporal drops in the temperature. Such coherent motions have been shown to be responsible for a very important fraction ( $75 \%$ and more) of the total turbulent fluxes (e.g., Gao et al. 1989) and therefore it is important that subgridscale models used in LES of the ABL can reproduce the relationship between the coherent structures and the dynamics of the nonresolved scales. Recently, some a priori studies that use conditional averaging techniques have addressed the importance of coherent structures on the SGS dissipation. Using data from filtered DNS of channel flow, Piomelli et al. (1996) indicated that backscatter of resolved kinetic energy tends to occur during sweeps, while positive SGS dissipation is associated with ejection events. Lin (1999) reexamines this issue based on analysis of resolved kinetic energy dissipation from LES with an eddy-viscosity model. He concludes that during ejections, both forward and backscatter occur, and the same is true during sweep events.

Porté-Agel et al. (1998, 2000b) used high-resolution temperature and velocity data collected in the surface layer with 3D sonic anemometers (a single anemometer in the first study and an array of six anemometers in the second study). These data were used to compute the 1D and 2D surrogates, respectively, of the SGS heat fluxes and SGS dissipation of the temperature variance. Using conditional averaging based on the temperature gradients, Porté-Agel et al. (1998, 2000b) concluded that during flow events associated with strong increments of vertical velocity (possibly associated with the onset of ejection events), negative values of the SGS dissipation have an important relative contribution regardless of atmospheric stability. Negative SGS dissipation is associated with the transfer of temperature variance from the small scales to the resolved field (backscatter). The onset of sweeps is associated with large positive values of the SGS dissipation.

In this paper, we use data collected with 12 3D sonic anemometers placed in two horizontal arrays in the surface layer to study the statistics of the SGS scalar flux and variance dissipation. We also test the ability of various SGS models to capture the characteristics of the SGS heat flux and dissipation measured in the field. Three models are studied: the eddy-diffusion model, the nonlinear (gradient) model, and a mixed model that is a linear combination of the eddy-diffusion model and the nonlinear model. The effect of the filter size on the results is also explored. We also study the relation between coherent structures of the flow and the SGS variables by continuing the use of two types of conditional sampling techniques applied to different variables. As in Porté-Agel et al. $(1998,2000 b)$ conditional averaging is first applied to the SGS variables based on the resolved temperature gradients, possibly associated with the onset of sweep and ejection events. Furthermore, conditional averaging is applied to the velocity and temperature fields measured with a vertical array of anemometers in order to capture the vertical distribution of coherent structures associated with strong positive and negative SGS dissipation.

\section{Experiment}

\section{a. Description of setups and datasets}

The data used in this study were collected in a field experiment carried out over a bare soil surface at the Campbell Tract research field of the University of California at Davis during the summer of 1999. The instruments were placed near the northeastern corner of a rectangular flat field of about $600 \mathrm{~m}$ (southward) $\times$ $300 \mathrm{~m}$ (westward) in order to guarantee a long homogeneous fetch in the upwind direction. The prevailing wind came from the southwest. The soil surface had furrows in the north-south direction, and the average furrow depth was $10 \mathrm{~cm}$.

The longitudinal $\left(u_{1}\right)$, lateral $\left(u_{2}\right)$, and vertical $\left(u_{3}\right)$ wind velocity components as well as the air temperature $(\theta)$ were measured simultaneously using 12 Campbell Scientific CSAT3 triaxial sonic anemometers (pathlength $=10 \mathrm{~cm}$ ). The sampling frequency was $20 \mathrm{~Hz}$. The supporting meteorological measurements included net radiation, water vapor concentration, relative humidity, and skin temperature.

The data analyzed here were obtained during five 30min periods in which the sensors were arranged differently. The arrangements are denoted as setups A, B, C, $\mathrm{D}$, and E, respectively. Two major arrangements of the sensors were used. The first arrangement corresponds to two parallel horizontal arrays of sensors (setups A, B, C, and D). A similar arrangement, also consisting of two horizontal arrays of anemometers, was used by Tong et al. (1999) to study the statistical nature of the SGS velocities and stresses. The second setup is a single vertical array of anemometers (setup E). The dates and starting times for the five periods are given in Table 1, together with details of the arrangement of the sensors and the turbulence and meteorological conditions. A description of the five setups is given below.

In the first four setups $(\mathrm{A}, \mathrm{B}, \mathrm{C}$, and $\mathrm{D})$, the sonic anemometers were placed in two parallel horizontal arrays aligned roughly perpendicular to the prevailing wind. The lower array has seven anemometers and the upper array has five anemometers, and they are located at heights (measured from the bottom of the furrows) $z_{1}=2.38 \mathrm{~m}$ and $z_{2}=2.88 \mathrm{~m}$, respectively. The distance between adjacent sensors in the same array, $d$, is different for the four setups (see Table 1). The filter sizes considered range from 1 to $7 \mathrm{~m}$. This range covers many regimes of interest in practical LES: for LES on domain 
TABLE 1. Summary of meteorological and turbulence conditions during the five measurement periods corresponding to different instrument setups: two horizontal parallel arrays were used in setups A, B, C, and D, while setup E consists of a vertical array.

\begin{tabular}{|c|c|c|c|c|c|}
\hline Meteological and turbulence conditions & Setup A & Setup B & Setup C & Setup D & Setup E \\
\hline Day & 9 Jun & 8 Jun & 14 Jun & 12 Jun & 19 Jun \\
\hline Starting time for the 30 -min period (LST) & 1730 & 1750 & 1755 & 1635 & 1840 \\
\hline Distance between adjacent sensors $(d)(\mathrm{m})$ & 0.20 & 0.40 & 0.82 & 1.42 & - \\
\hline Angle $\beta\left(^{\circ}\right)$ & +5.4 & -1.7 & +5.3 & +5.5 & - \\
\hline Filter size $\Delta(\mathrm{m})$ & 1.00 & 2.02 & 4.07 & 7.08 & 2.02 \\
\hline Mean horizontal wind speed $\left(\left\langle u_{1}\right\rangle\right)\left(\mathrm{m} \mathrm{s}^{-1}\right)$ & 4.69 & 4.67 & 5.32 & 4.70 & 3.14 \\
\hline Mean air temperature $(\langle\theta\rangle)(\mathrm{K})$ & 299.9 & 297.7 & 301.9 & 303.8 & 302.6 \\
\hline Rms vertical velocity $\left(\sigma_{u 3}\right)\left(\mathrm{m} \mathrm{s}^{-1}\right)$ & 0.36 & 0.35 & 0.40 & 0.39 & 0.33 \\
\hline Rms temperature $\left(\sigma_{\theta}\right)(\mathrm{K})$ & 0.61 & 0.57 & 0.72 & 0.98 & 0.66 \\
\hline Friction velocity $\left(u_{*}\right)\left(\mathrm{m} \mathrm{s}^{-1}\right)$ & 0.24 & 0.24 & 0.29 & 0.24 & 0.23 \\
\hline Streamwise heat flux $\left.\left(u_{1}^{\prime} \theta^{\prime}\right\rangle\right)\left(\mathrm{K} \mathrm{m} \mathrm{s}^{-1}\right)$ & -0.225 & -0.237 & -0.348 & -0.297 & -0.163 \\
\hline Vertical heat flux $\left(\left\langle u_{3}^{\prime} \theta^{\prime}\right\rangle\right)\left(\mathrm{K} \mathrm{m} \mathrm{s}^{-1}\right)$ & +0.093 & +0.076 & +0.125 & +0.182 & 0.071 \\
\hline Obukhov length $(L)(\mathrm{m})$ & -10.80 & -13.7 & -15.7 & -6.01 & -13.15 \\
\hline Stability parameter $(z / L)$ & -0.22 & -0.17 & -0.15 & -0.35 & -0.14 \\
\hline
\end{tabular}

lengths of several kilometers using several hundreds of grid points, one uses grid sizes on the order of $10 \mathrm{~m}$ (as in setup D). There are also many applications in the literature where the computational domain is only a fraction of the boundary layer height (e.g., Shaw and Schumann 1992; Patton et al. 1998; Su et al. 1998), with grid sizes of a few meters (as in setups A and B). Our fine-grid results are also directly relevant to LES using nested grids (e.g., Khanna and Brasseur 1998). The vertical separation distance of the arrays influences the accuracy of vertical derivatives of filtered variables. Mainly for reasons of experimental practicality, we choose a fixed distance. Changing the height as well as probe spacings would have greatly complicated the field ex- periments. The vertical separation distance chosen (equal to $0.5 \mathrm{~m}$ ) is smaller, but of the same order of magnitude, than the smallest filter size considered (setup A). One limitation of this choice is that for the case of setup $\mathrm{D}(\Delta=7 \mathrm{~m})$, vertical derivatives will be evaluated at a significantly finer resolution than the filter scale (or the corresponding LES mesh spacing). However, since the variables being differentiated are already filtered at larger scales, it is assumed that the separation distance has little impact on the results.

Figures 1a-c show a sketch of the relative position of the anemometers. The reference system is chosen so that $i=1,2,3$ corresponds to the streamwise, spanwise, and vertical directions, respectively. Since the spanwise (a)

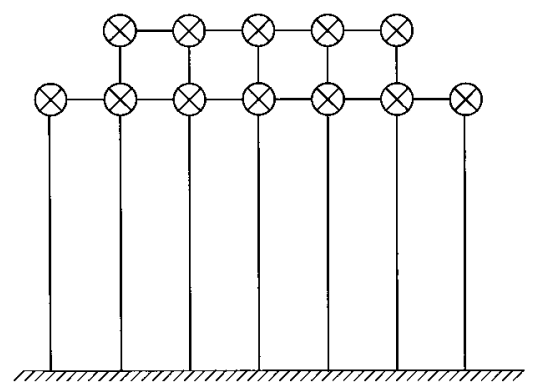

(b)

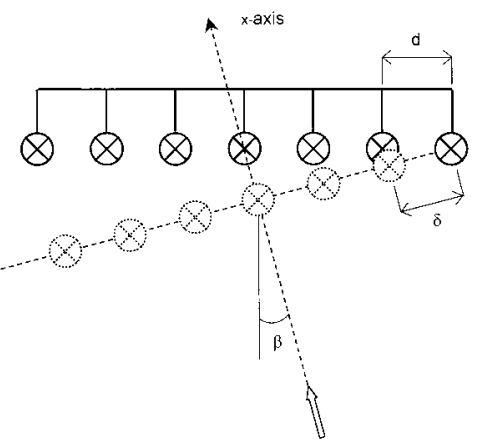

(c)

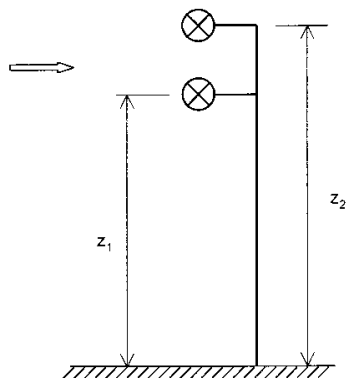

FIG. 1. Sketch of the instrument setup: (a) front view, (b) top view, and (c) lateral view. 
(a)

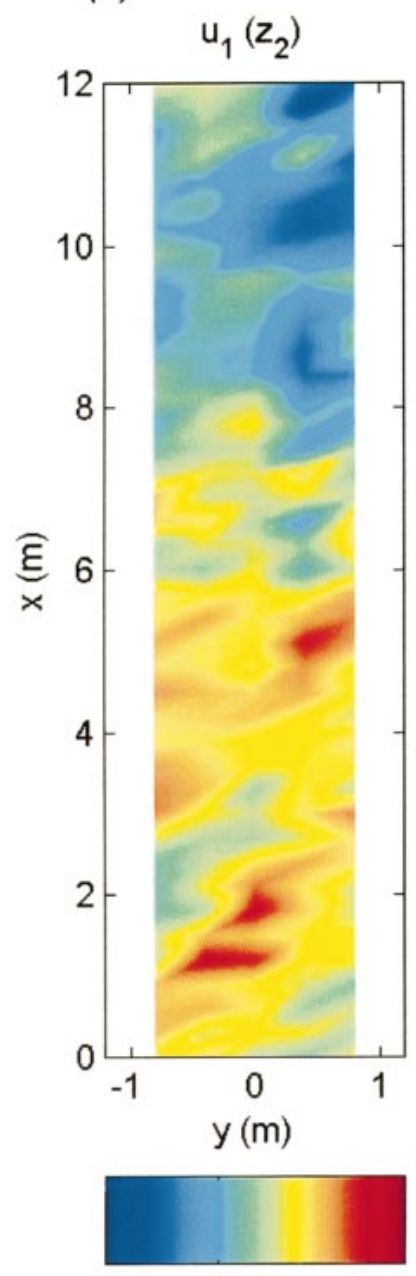

5 (b)

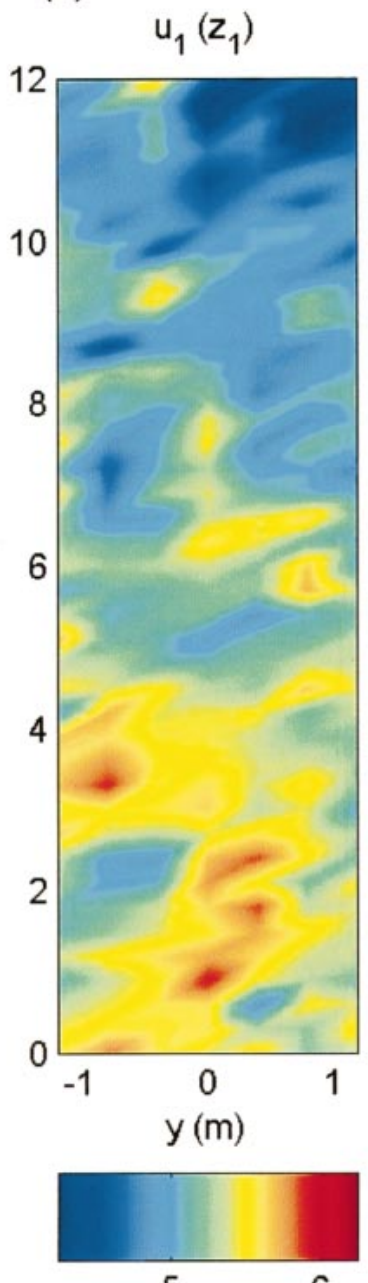

(c)

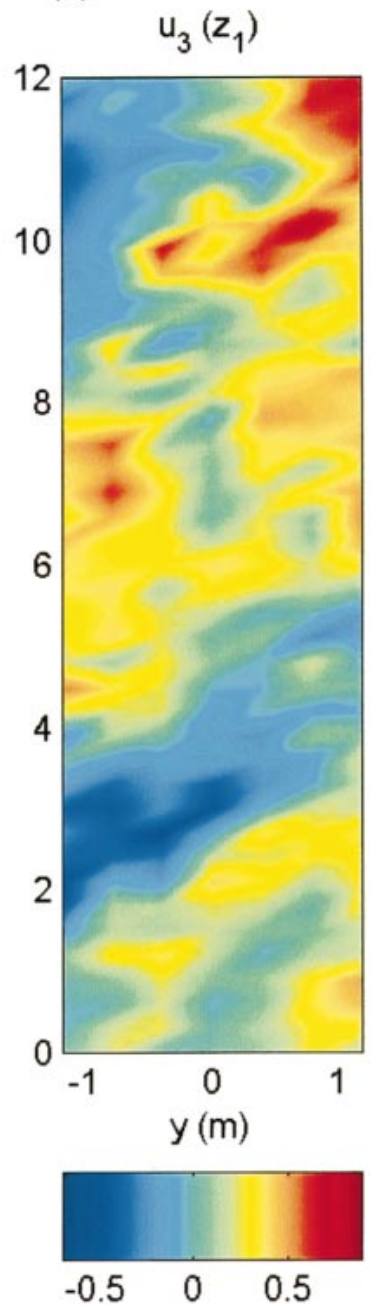

(d)

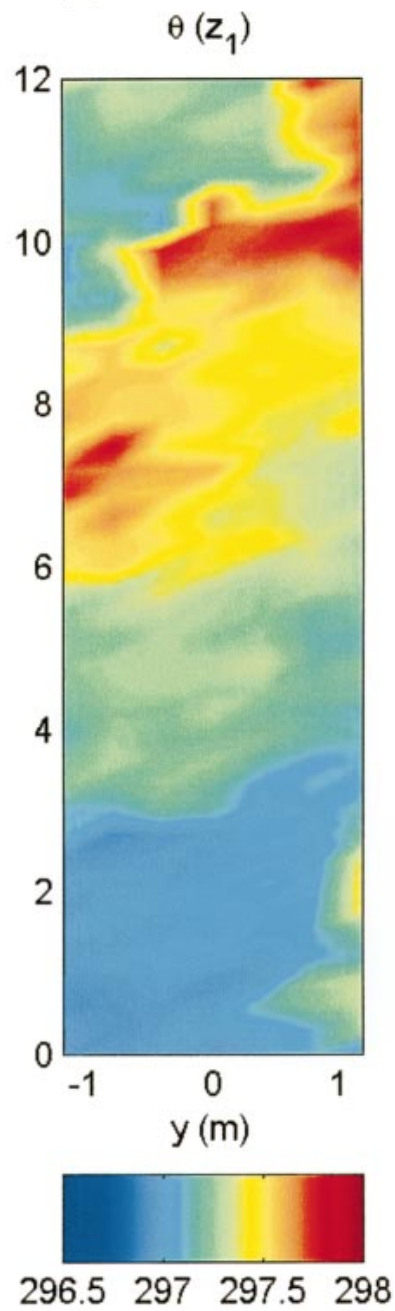

FIG. 2. Contour plots in horizontal planes of the streamwise velocity $\left(u_{1}\right.$ in $\left.\mathrm{m} \mathrm{s}^{-1}\right)$ at the two heights $\left(z_{1}\right.$ and $\left.z_{2}\right)$, vertical velocity $\left(u_{3}\right.$ in $\left.\mathrm{m} \mathrm{s}^{-1}\right)$ at $z=z_{1}$, and temperature $(\theta$ in $\mathrm{K})$ also at $z=z_{1}$.

direction (perpendicular to the direction of the mean wind for each period) does not correspond exactly with the direction of alignment of the sensors, a shift is applied to the time series based on the use of Taylor's frozen flow hypothesis in order to have simultaneous readings in the positions corresponding to the $x$ axis (dotted line in Fig. 1b). Therefore, the distance between two consecutive sensors in the $x$ direction is $\delta=d \times$ $\cos \beta$, where $\beta$ is the angle between the direction of the mean wind and the direction perpendicular to the sensors. The values of $\beta$ and $d$ corresponding to the four periods (A, B, C, and D) are given in Table 1. The momentum roughness length $z_{o}$ is $2 \mathrm{~mm}$. Note that the four periods were selected to have similar meteorological and turbulence conditions (Table 1) in order to better isolate the effect of the arrangement of the sensors on the results. Particularly, the wind direction and intensity are very similar, and the stability parameter $z / L$ falls between -0.15 and -0.35 for the four periods, indi- cating unstable conditions. Here $L$ is the Obukhov length,

$$
L=\frac{-u_{*}^{3} \rho}{k g\left[\frac{H}{C_{p}\langle\theta\rangle}+0.61 E\right]},
$$

where $u_{*}\left[=(\tau / \rho)^{1 / 2}=\left(-\left\langle u_{1}^{\prime} u_{3}^{\prime}\right\rangle\right)^{1 / 2}\right]$ is the friction velocity, $\tau$ is the surface shear stress, $\rho$ is the density of air, $H$ is the sensible heat flux, $\langle\theta\rangle$ is the mean air temperature, $E$ is the water vapor flux $\left(E=\rho\left\langle u_{3}^{\prime} q^{\prime}\right\rangle\right), q^{\prime}$ is the fluctuation in the water vapor concentration, $g$ is the gravitational acceleration, and $k(=0.4)$ is von Kármán's constant. Setup B is used as a base case to study the measured and modeled SGS heat fluxes and SGS dissipation of temperature variance (section 3), as well as their relation to coherent structures of the flow (section 4a). In addition, setups A, C, and D are also used to 


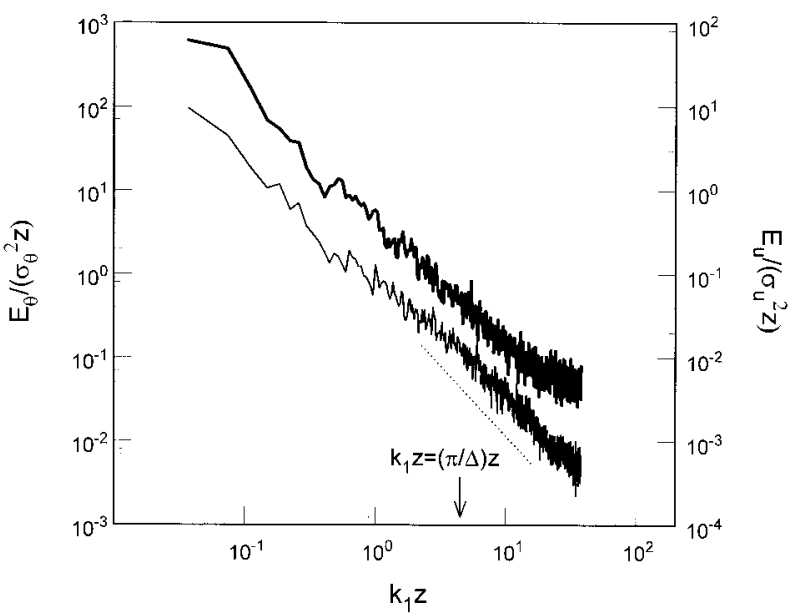

FIG. 3. Normalized power spectra for the streamwise velocity (thick line) and the temperature (light line) for measurement period B. The $-5 / 3$ power law is also shown (dotted line). The arrow shows $\mathrm{k}_{1} z$ corresponding to the filter scale $\Delta$ (see section $3 a$ ).

explore the effect of filter size (which depends on $d$ as explained in section 3a) on the results.

Setup E consists of a vertical array with the $123 \mathrm{D}$ sonic anemometers placed at heights (measured from the bottom of the furrows) $z_{(1)}=0.52 \mathrm{~m}, z_{(2)}=0.77 \mathrm{~m}$, $z_{(3)}=1.02 \mathrm{~m}, z_{(4)}=1.27 \mathrm{~m}, z_{(5)}=1.53 \mathrm{~m}, z_{(6)}=1.78$ $\mathrm{m}, z_{(7)}=2.03 \mathrm{~m}, z_{(8)}=2.28 \mathrm{~m}, z_{(9)}=2.79 \mathrm{~m}, z_{(10)}=$ $3.31 \mathrm{~m}, z_{(11)}=3.81 \mathrm{~m}$, and $z_{(12)}=4.77 \mathrm{~m}$, respectively.
Note that the parentheses in the subscripts indicate that the heights correspond to the vertical setup (as opposed to the double horizontal array). From Table 1, measurement period $\mathrm{E}$ also corresponds to unstable conditions $(z / L=-0.14)$. Data collected with setup $E$ are used in section $4 d$ to study the vertical distribution of coherent structures of the flow during strong positive and negative dissipation events.

\section{b. Sample raw data and longitudinal spectra}

In Fig. 2, 2D color contour plots of the streamwise velocity $\left(u_{1}\right)$ at the two heights $\left(z_{1}\right.$ and $\left.z_{2}\right)$, as well as of the vertical velocity $\left(u_{3}\right)$ and temperature $(\theta)$ at the lower level $\left(z_{1}\right)$, are shown corresponding to the measurement period $\mathrm{B}$. The short axis corresponds to the direction perpendicular to the main wind (nearly parallel to the sensors) ( $y$ direction). The longer axis corresponds to the direction of the main wind ( $x$ direction) and is obtained using Taylor's frozen flow hypothesis to convert the time coordinate into a space coordinate.

In Fig. 2 the presence of structures of the flow can be identified as well as their relative size and horizontal dimensions. Note that their relative size is comparable and smaller than the filter size $\Delta$ (to be identified later with a multiple of the distance among sensors, $d$ ), which clearly indicates that they have an important influence on the dynamics of the SGS variables. There is clear evidence of the negative correlation between the streamwise and
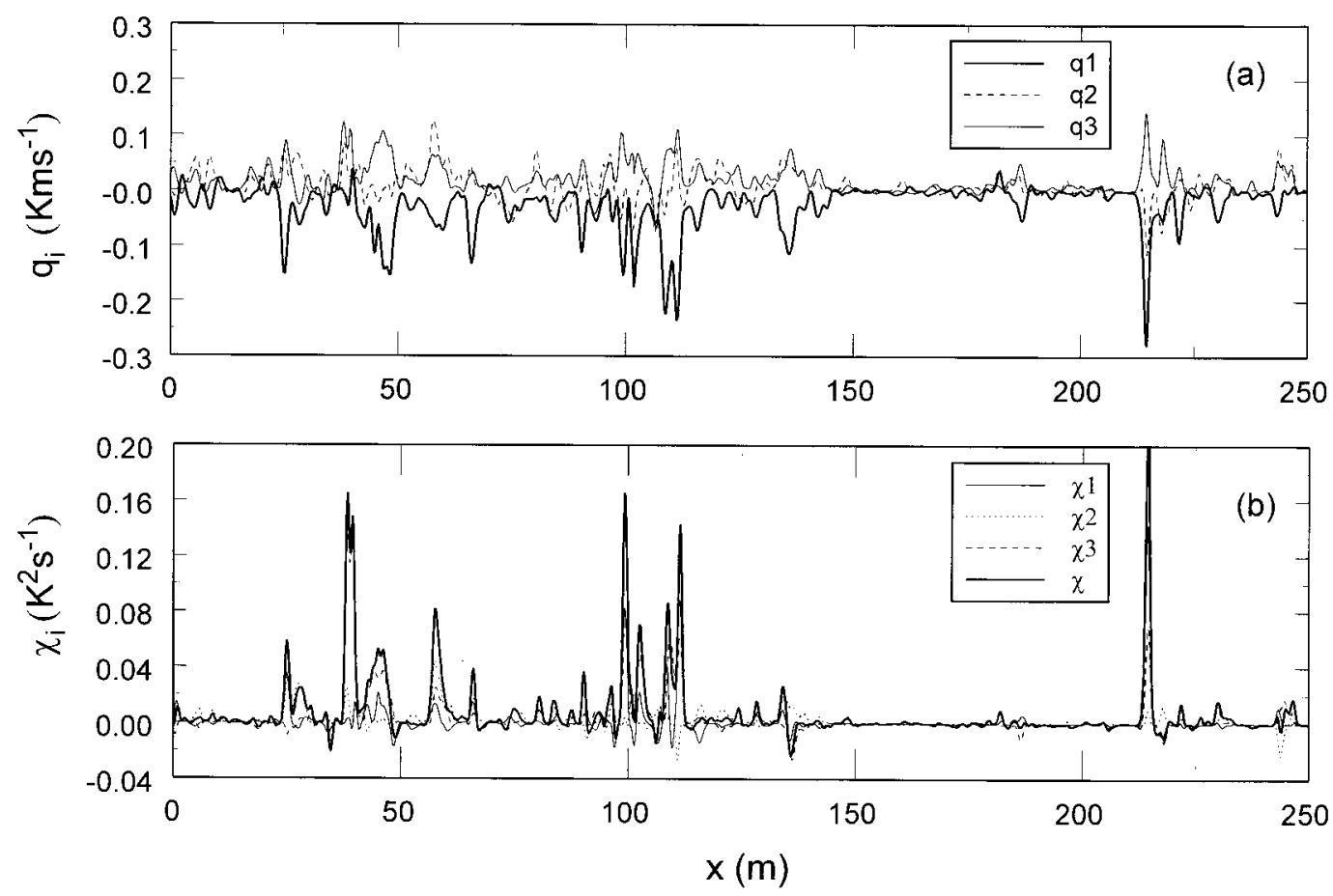

FIG. 4. (a) Portion of the three components of the SGS heat flux signals: streamwise $q_{1}$, spanwise $q_{2}$, and vertical $q_{3}$. (b) Portion of the surrogates of the three components of the SGS dissipation of the temperature variance: streamwise $\chi_{1}$, spanwise $\chi_{2}$, vertical $\chi_{3}$, and total $\chi=\chi_{1}+\chi_{2}+\chi_{3}$. 
vertical velocity fluctuations $\left(\left\langle u_{1}^{\prime} u_{3}^{\prime}\right\rangle<0\right)$ in wall-bounded turbulent flows. A deceleration of the flow in the streamwise direction $\left(u_{1}^{\prime}<0\right)$ is associated with an upward motion or ejection of the air $\left(u_{3}^{\prime}>0\right)$ from below. Under unstable conditions, these ejections carry relatively warm air upward producing an increase in the temperature $\left(\theta^{\prime}\right.$ $>0)$.

The power spectra for the streamwise velocity and temperature signals measured at height $z=z_{1}(=2.38 \mathrm{~m})$ during the measurement period $\mathrm{B}$ are computed and presented in Fig. 3. They are obtained from 25 segments of 1024 points each, using a Bartlett window (Press et al. 1992). In Fig. 3 the power spectra ( $E_{k}$ for a generic signal $k$ ) are normalized as $E_{k} / \sigma_{k}^{2} z$, where $\sigma_{k}^{2}$ is the variance of the signal. The wavenumber $k_{1}$ is based on the sampling frequency, using Taylor's hypothesis, and it is normalized by $z$. The slope of the velocity spectrum is $-5 / 3$ for a wide range of wavenumbers, indicative of the inertial subrange. The slope is smaller for smaller wavenumbers.

\section{c. Experimental error}

The sonic anemometer's specifications are that the least significant bits of the digital readings correspond to 0.25 to $2 \mathrm{~mm} \mathrm{~s}^{-1}$ for velocity and $0.002^{\circ} \mathrm{C}$ for temperature. The actual specified experimental uncertainty in their analog output is $0.015 \mathrm{~m} \mathrm{~s}^{-1}$ for the streamwise and spanwise velocities, $0.004 \mathrm{~m} \mathrm{~s}^{-1}$ for the vertical velocity, and $0.026^{\circ} \mathrm{C}$ for temperature. This error corresponds to about $2 \%$ (for velocity) and $4 \%$ (for temperature) of the corresponding typical rms values. At the smallest filter scale used $(\Delta=1 \mathrm{~m})$, assuming inertial range scalings, we estimate typical velocity increments to be of the order $\delta u_{\Delta} \sim 0.2 \mathrm{~m} \mathrm{~s}^{-1}$ and temperature decrements $\delta \theta_{\Delta} \sim 0.15^{\circ} \mathrm{C}$. Hence, the relative error at the scale $\Delta$ is about $7 \%$ for velocities and $17 \%$ for temperatures. For the product $\left(\delta u_{\Delta} \delta \theta_{\Delta}\right.$, which is a scaling surrogate for the SGS heat flux), the relative error is about $7+17 \sim 24 \%$. A reasonable error estimate for the SGS heat flux measured from our sonic anemometers is thus between $20 \%$ and $25 \%$. At larger filter scales the fluxes increase and hence the relative errors are smaller. This estimate is based on unbiased errors. However, for each sonic there is also the possibility of a biased error, which can shift the mean value of the signal but leave fluctuating values within the specified errors. Indeed, we found differences of mean values among transverse sensors that are larger than the 0.02 $\mathrm{m} \mathrm{s}^{-1}$ or $0.02^{\circ} \mathrm{C}$ errors specified by the manufacturers. In particular, two sensors showed significantly larger deviations in mean. Hence, we have subtracted the mean value from the readings of these two sensors whose mean differed most from the others. The impact of this biased error is not large, however, as later it will be shown that the impact of the mean gradients on mean SGS dissipation is on the order of 5\% only, that is, within the relative error estimated above.

Another source of error is path averaging of the in- 

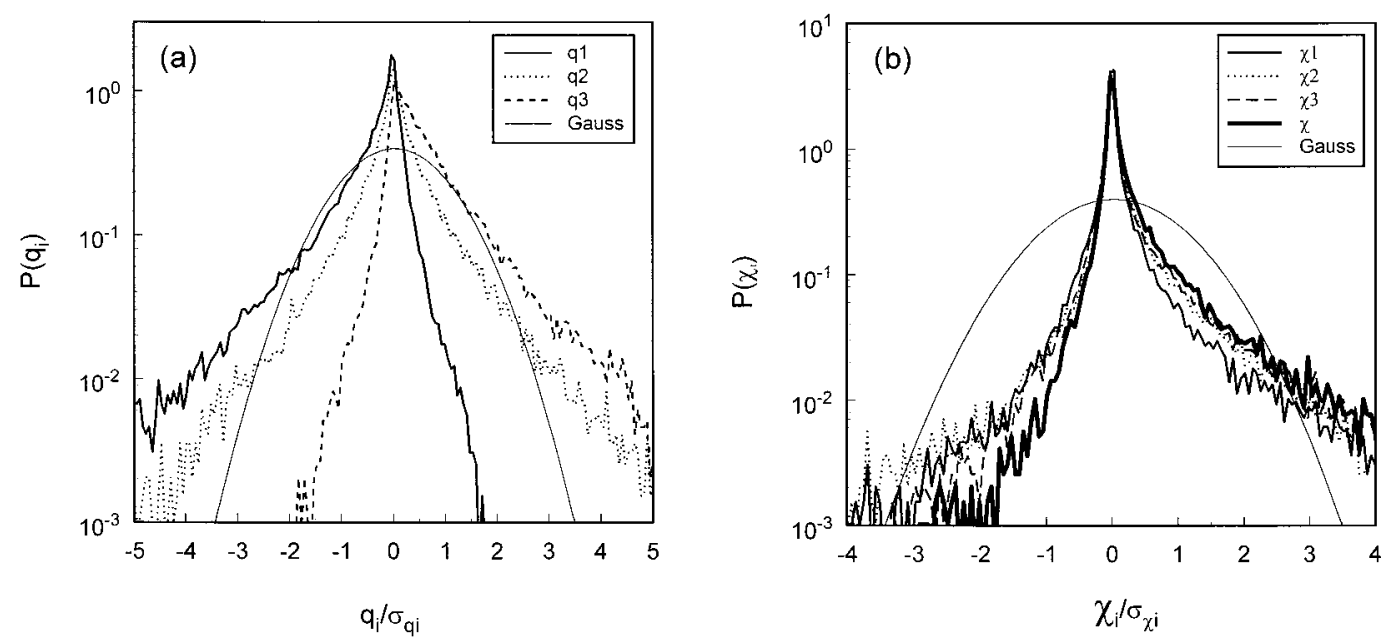

FIG. 5. (a) Probability density function (PDF) of the three components of the SGS heat flux (streamwise $q_{1}$, spanwise $q_{2}$, and vertical $q_{3}$ ) normalized with their standard deviation. (b) PDF of the surrogates of the SGS dissipation of the temperature variance (streamwise $\chi_{1}$, spanwise $\chi_{2}$, vertical $\chi_{3}$, and overall $\chi=\chi_{1}+\chi_{2}+\chi_{3}$ ). The thin solid line is the Gaussian distribution with zero mean.

dividual sonic anemometers. The path-averaging scale is about $10 \mathrm{~cm}$; that is, we may consider that the signals are being spatially "prefiltered" at a scale $\Delta / 10$ (for the setup A) before being filtered at scale $\Delta$ for the present analysis. The dominant contributions to the SGS fluxes come from scales that are in the vicinity of $\Delta$ [mostly from the neighboring octave (see, e.g., Meneveau and Katz 2000)], and not from scales that are much smaller. We have checked this by additionally prefiltering the measured signals in time at even larger scales (e.g., at scale $\Delta / 3$ ). The SGS fluxes evaluated from such prefiltered signals are almost identical to the ones evaluated from the original signals. It is inferred that the path averaging at $10 \mathrm{~cm}$ has no appreciable effect on the presently studied SGS variables at scales $1 \mathrm{~m}$ and above.

Finally, flow distortion is another potential source of error. We have not performed an exhaustive analysis of flow distortion. However, we expect this source of error to be small since the actual blocked area associated with the anemometers is only a very small fraction of the projected area of the flow as it traverses the array.

\section{Measured and modeled SGS heat fluxes and SGS dissipation of temperature variance}

\section{a. Measured SGS variables}

Each component of the SGS heat flux, $q_{i}$, is computed according to its definition [Eq. (1.1)] $q_{i}=\widetilde{u_{i}} \theta-\tilde{u_{i}} \tilde{\theta}$. As in Porté-Agel et al. (2000b), a 2D filter of size $\Delta$ is used, which consists of the combination of a Gaussian filter in the streamwise direction and a box filter in the direction parallel to the sensors. There are various filters usually used in LES and a priori studies [the spectral cutoff filter, the box filter, and the Gaussian filter (see, e.g., Piomelli et al. 1988)]. Many LES codes for ABL use spectral methods in planes parallel to the ground for which a spectral cutoff filtering would seem most appropriate for the a priori analysis. However, as summarized in Meneveau and Katz (2000), when filtering spatially localized phenomena such as sharp gradients (e.g., those associated with the onset of ejection and sweep events), the spectral cutoff filter produces "ringing effects" due to the Gibbs phenomenon with spatially nonlocal impact. Moreover, due to its slow $x^{-1}$ decay in physical space, this filter shape is very difficult to approximate with a practically feasible number of sensors. For instance, in their analysis Tong et al. (1999) use a spectral filter in the streamwise direction, but in the cross-stream direction, their filter's transfer function, shown in their Fig. 3a, resembles that of a box filter more than it does that of a sharp spectral cutoff filter, especially at wavenumbers smaller than the filter wavenumber $\pi / \Delta$. In this regard, their analysis can be considered effectively a quasi-mixed box filtering in the crossstream direction and spectral filtering in the streamwise direction.

Due to these difficulties, we prefer to remain fully within the context of spatially localized filters, which can be well represented by the experimental arrangement (but recognizing the less precise correspondence to spectral-based LES). As shown in Cerutti and Meneveau (2000) in the context of arrays of hot-wire sensors for similar studies in laboratory turbulence, the box filter can be well represented by a finite number of sensors. To increase smoothness we use a Gaussian filter in the streamwise direction. This filter is very similar in many respects to the box filter although it is smoother since it is more localized also in spectral space. In order to assess the sensitivity of the results to the filter type, other filters have been used in the streamwise direction, including spectral cutoff and tophat filters. No significant qualitative differences have been found with respect to the results obtained with the Gaussian filter presented below. The SGS heat fluxes and dis- 
sipation are computed in the transverse position $y=0$ and the vertical position $z=z_{1}$.

For a generic variable $\alpha(x, y, z)$, where $x, y$, and $z$ are the streamwise $\left(x_{1}\right)$, spanwise $\left(x_{2}\right)$, and vertical $\left(x_{3}\right)$ spatial coordinates, respectively, the filtered variable $\tilde{\alpha}\left(x, y_{J}, z_{K}\right)$, corresponding to the lateral position $y_{J}$ and height $z_{K}$, is computed as follows: the box filter applied in the spanwise direction consists of the average $\bar{\alpha}\left(x, y_{J}, z_{K}\right)=1 / N \sum_{j=1}^{N}$ $\alpha\left(x, y_{j}, z_{K}\right)$, where $y_{j}$ is the lateral position of each of the $N$ sensors centered around the ordinate $y_{J}$. Here $N$ was chosen to be 5 in order to maximize the number of sensors used to filter in the spanwise direction. For the dataset B, this corresponds to a filter size $\Delta=5 \times \delta=2.0 \mathrm{~m}$. Then $\bar{\alpha}\left(x, y_{J}, z_{K}\right)$ is filtered in the streamwise direction by convolution with a filter function $G_{\Delta}: \tilde{\alpha}\left(x, y_{J}, z_{K}\right)=\int \bar{\alpha}(x$, $\left.y_{J}, z_{K}\right) G_{\Delta}\left(x-x^{\prime}\right) d x^{\prime}$, where $x^{\prime}$ is an integration variable. For computational convenience the filtering is done in wave space using the fast Fourier transform (Press et al. 1992). The results presented here were obtained using a Gaussian filter whose Fourier transform is of the form: $\hat{G}_{\Delta}=\exp \left[-\left(k_{1}^{2} \Delta^{2} / 24\right)\right]$, where $k_{1}$ is the wavenumber and $\Delta$ the filter size. The filter size was chosen to be $\Delta=5$ $\times \delta$, equal to the filter size in the transverse direction. As shown in Fig. 3, the selected filter size (wavenumber $k_{1}$ $=\pi / \Delta$ ) falls well into the inertial subrange, characterized by the $-5 / 3$ slope in the spectra.

The use of Taylor's hypothesis in the inertial subrange is well accepted in the atmospheric surface layer for turbulent intensities (rms to mean streamwise velocity ratio) of up to 30\% and more (e.g., Peltier et al. 1996; Kiely et al. 1996; Katul et al. 1977a; Wyngaard and Clifford 1977). This value is well above the turbulent intensities found in our measurement periods (see Table 1). In particular, the use of Taylor's hypothesis to approximate streamwise filtering with time filtering was studied by Tong et al. (1998), using data from a LES of the ABL. They concluded that although the fluctuations in the convection velocity have some effect on the computed SGS fluxes and stresses due to the change in the effective filter size $\Delta$ and shape, the sensor array is a feasible technique for SGS measurement in the atmospheric surface layer.

Figure 4 shows signals of SGS heat flux and dissipation. Figure 4a shows a segment of the three components of the measured SGS heat flux $q_{i}$. The signals have a strong degree of intermittency, and $q_{1}$ and $q_{3}$ appear to be strongly anticorrelated. Note that the magnitude of the mean values $\left\langle q_{1}\right\rangle$ and $\left\langle q_{3}\right\rangle$, presented in Table 2, are comparable, while $\left\langle q_{2}\right\rangle$ is much smaller. Figure 5a presents the probability density function (PDF) of the measured SGS heat fluxes. The fluxes show a clear non-Gaussian behavior, with strong asymmetry in the PDFs of $q_{1}$ and $q_{3}$, and exponential tails. This is consistent with the results obtained by Porté-Agel et al. (2000b).

The three components of the SGS dissipation of temperature variance, $\chi_{1}, \chi_{2}$, and $\chi_{3}$, corresponding to the streamwise, spanwise, and vertical directions, respectively, are computed according to their definition $\chi_{m}=$ $-q_{m}\left(\partial \tilde{\theta}^{\prime} / \partial x_{m}\right)$ (no summation over $m$ ) at the transverse position $y_{J}=0$ and height $z_{K}=z_{1}$. In the spanwise direction, the derivative involves the difference between the filtered values at $y_{J}=+\delta$ and $y_{J}=-\delta$. In the streamwise direction derivatives are computed from time derivatives invoking Taylor's hypothesis. Center difference approach is employed with the values of the filtered variables at positions $x=+\Delta / 2$ and $x=-\Delta /$ $2\left(y_{J}=0\right)$. Vertical derivatives are computed using a one-sided finite-difference approach with the values of the filtered variables at the vertical positions $z=z_{2}$ and $z=z_{1}$. The total SGS dissipation of temperature variance $\chi$ is also obtained by adding the three components $\left(\chi=\chi_{1}+\chi_{2}+\chi_{3}\right)$. Figure $4 \mathrm{~b}$ shows a segment of the three components and the overall value of the measured SGS dissipation of resolved temperature variance. The signals have a very strong degree of intermittency and all four of them have occasional negative values, indicating backscatter, that is, transfer of temperature variance from the small scales to the large scales. The PDF of the measured SGS dissipation, shown in Fig. 5b, is also very different from the Gaussian distribution. The strong asymmetry shows that the positive average value of $\chi$ (forward cascade of scalar variance in the mean) is due to the fact that strong positive values occur more frequently, and are stronger in magnitude, than negative values.

\section{b. Modeled SGS variables}

In this section, the SGS heat flux is modeled for the case of setup B (same as in section 3a) using the three models presented in section 1a: the eddy-diffusion model [Eq. (1.3)], the nonlinear model [Eq. (1.5)], and the mixed model [Eq. (1.6)]. Spatial derivatives of the filtered variables are needed for all three models. They are computed in the same manner as the temperature gradients in section $3 \mathrm{a}$.

The model coefficients for the eddy-diffusion and nonlinear models, $\operatorname{Pr}_{T}^{-1} C_{S}^{2}$ and $C_{\mathrm{nl}}$, respectively, are computed to guarantee that the mean modeled SGS dissipation matches the mean measured dissipation, that is, $\left\langle\chi^{\mathrm{mod}}\right\rangle=$ $\langle\chi\rangle$. The coefficients obtained using this criterion are

$$
\begin{aligned}
\left(\operatorname{Pr}_{T}^{-1} C_{S}^{2}\right) & =\frac{-\left\langle q_{i} \frac{\partial \tilde{\theta}^{\prime}}{\partial x_{i}}\right\rangle}{\left\langle\Delta^{2}|\tilde{S}| \frac{\partial \tilde{\theta}}{\partial x_{i}} \frac{\partial \tilde{\theta}^{\prime}}{\partial x_{i}}\right\rangle}, \quad \text { and } \\
\left(C_{\mathrm{nl}}\right) & =\frac{\left\langle q_{i} \frac{\partial \tilde{\theta}^{\prime}}{\partial x_{i}}\right\rangle}{\left\langle\Delta^{2} \frac{\partial \tilde{u}_{i}}{\partial x_{k}} \frac{\partial \tilde{\theta}}{\partial x_{k}} \frac{\partial \tilde{\theta}^{\prime}}{\partial x_{i}}\right\rangle} .
\end{aligned}
$$

Note that the Smagorinsky coefficient $\left(C_{S}\right)$ comes from the eddy-viscosity model for the SGS stresses $\tau_{i j}=\widetilde{u_{i} u_{j}}-\widetilde{u}_{i} \tilde{u}_{j}$ in the filtered momentum equation. 
In particular, the formulation of the eddy-viscosity model is $\tau_{i j}^{\mathrm{ev}}=-2 C_{S}^{2} \Delta^{2}|\tilde{S}| \tilde{S}_{i j}$. The contribution of the subgrid scales on the level of fluctuation of the resolved turbulent kinetic energy (TKE) is given by the SGS dissipation of resolved TKE, $\Pi$ (analogous to $\chi$ for the resolved temperature variance), which is defined as $\Pi=-\tau_{i j} \tilde{S}_{i j}$. The value of the coefficient $C_{S}$ can be calculated by forcing the modeled SGS dissipation of resolved TKE $\left\langle\Pi^{\mathrm{ev}}\right\rangle=-\left\langle\tau_{i j}^{\mathrm{ev}} \bar{S}_{i j}\right\rangle$ to match the mean measured SGS dissipation $\langle\Pi\rangle$. This yields the estimation

$$
C_{S}^{2}=\frac{-\left\langle\tau_{i j} \tilde{S}_{i j}\right\rangle}{\left\langle 2 \Delta^{2}|\tilde{S}| \tilde{S}_{i j} \tilde{S}_{i j}\right\rangle} .
$$

See O'Neil and Meneveau (1997) for more details.

In order to estimate the value of the model coefficients for the mixed model, $\left[\operatorname{Pr}_{T}^{-1} C_{S}^{2}\right]^{*}$ and $\left[C_{n l}\right]^{*}$, we need to use an additional condition (besides the match of the mean measured and modeled SGS dissipation). The second condition is based on scale similarity arguments applied to the SGS fluxes, as presented in detail in the appendix. The mixed model coefficients obtained applying these criteria are

$$
\begin{aligned}
{\left[C_{\mathrm{nl}}\right]^{*}=} & \frac{\left\langle M_{i} N_{i}\right\rangle\left\langle q_{i} \frac{\partial \bar{\theta}^{\prime}}{\partial x_{i}}\right\rangle+\left\langle L_{i} N_{i}\right\rangle\left\langle\Delta^{2}|\tilde{S}| \frac{\partial \bar{\theta}}{\partial x_{i}} \frac{\partial \bar{\theta}^{\prime}}{\partial x_{i}}\right\rangle}{\left\langle N_{i} N_{i}\right\rangle\left\langle\Delta^{2}|\bar{S}| \frac{\partial \bar{\theta}}{\partial x_{i}} \frac{\partial \bar{\theta}^{\prime}}{\partial x_{i}}\right\rangle+\left\langle M_{i} N_{i}\right\rangle\left\langle\Delta^{2} \frac{\partial \bar{u}_{i}}{\partial x_{k}} \frac{\partial \bar{\theta}}{\partial x_{k}} \frac{\partial \bar{\theta}^{\prime}}{\partial x_{i}}\right\rangle}, \text { and } \\
{\left[\operatorname{Pr}_{T}^{-1} C_{S}^{2}\right]^{*}=} & \frac{\left[C_{\mathrm{nl}}\right]^{*}\left\langle\Delta^{2} \frac{\partial \bar{u}_{i}}{\partial x_{k}} \frac{\partial \bar{\theta}}{\partial x_{k}} \frac{\partial \bar{\theta}^{\prime}}{\partial x_{i}}\right\rangle-\left\langle q_{i} \frac{\partial \bar{\theta}^{\prime}}{\partial x_{i}}\right\rangle}{\left\langle\Delta^{2}|\bar{S}| \frac{\partial \bar{\theta}}{\partial x_{i}} \frac{\partial \bar{\theta}^{\prime}}{\partial x_{i}}\right\rangle},
\end{aligned}
$$

where

$$
\begin{aligned}
& L_{i}=\widehat{\bar{u}_{i} \bar{\theta}}-\hat{\bar{u}}_{i} \hat{\bar{\theta}}, \\
& M_{i}=\Delta^{2}\left(\widehat{|\bar{S}| \frac{\partial \bar{\theta}}{\partial x_{i}}}-\gamma^{2}|\hat{\bar{S}}| \frac{\partial \hat{\bar{\theta}}}{\partial x_{i}}\right), \quad \text { and } \\
& N_{i}=\Delta^{2}\left(\gamma^{2} \frac{\partial \hat{\bar{u}}_{i}}{\partial x_{k}} \frac{\partial \hat{\bar{\theta}}}{\partial x_{k}}-\frac{\partial \overline{\bar{u}}_{i} \frac{\partial \bar{\theta}}{\partial x_{k}} \frac{\partial x_{k}}{k}}{\text {. }} .\right.
\end{aligned}
$$

This procedure involves using two filters of sizes $\bar{\Delta}$ and $\hat{\Delta}$, with $\bar{\Delta}<\hat{\Delta}$. Note that the test filter of size $\hat{\Delta}$ is applied to the variables filtered at scale $\bar{\Delta}$. Given the arrangement of the sensors and the fact that a box filter is used in the direction parallel to the sensors, we find it optimal to use $\bar{\Delta}=3 \times \delta$ and $\hat{\Delta}=\Delta=5 \times \delta$. The first filter (at scale $\bar{\Delta}$ ) is performed using the $2 \mathrm{D}$ filter presented above (combination of Gaussian filter in the streamwise direction and box filter in the spanwise direction), but with a filter size $\bar{\Delta}=3 \times \delta$. Since the second filter at scale $\hat{\Delta}$ is applied to the variables obtained at a resolution $\bar{\Delta}$, we compute the test-filtered variable $\overline{\tilde{\alpha}}\left(x, y_{J}, z\right)$ using a box filter of the form $\overline{\tilde{\alpha}}(x$, $\left.y_{J}, z_{K}\right)=1 / 4\left[\tilde{\alpha}\left(x-\bar{\Delta} / 4, y_{J}-\delta, z_{K}\right)+\tilde{\alpha}\left(x+\bar{\Delta} / 4, y_{J}-\right.\right.$ $\left.\left.\delta, z_{K}\right)+\tilde{\alpha}\left(x-\bar{\Delta} / 4, y_{J}+\delta, z_{K}\right)+\tilde{\alpha}\left(x+\bar{\Delta} / 4, y_{J}+\delta, z_{K}\right)\right]$. The values of the coefficients obtained for the three models are $\operatorname{Pr}_{T}^{-1} C_{S}^{2}=0.019, C_{\mathrm{nl}}=0.34,\left[\operatorname{Pr}_{T}^{-1} C_{S}^{2}\right]^{*}=$ 0.0052 , and $\left[C_{\mathrm{nl}}\right]^{*}=0.24$. From the balance of the mean and modeled SGS dissipation of resolved TKE, we estimate [Eq. (3.3)] $C_{S}=0.090$. Also an estimate for $\operatorname{Pr}_{T}$ can be made through $C_{S}^{2} / \operatorname{Pr}_{T}^{-1} C_{S}^{2}=0.43$. These results are quite similar to previously reported values of $C_{S} \sim 0.09$ (e.g., Deardorff 1971; Piomelli et al. 1988), $\operatorname{Pr}_{T} \sim 0.4$ (e.g., Mason and Derbyshire 1990; Moin et al. 1991), and $C_{\mathrm{nl}} \sim 0.3$ [Meneveau and Katz (1999a,b) in the context of the momentum equation].

In Figs. 6b-d a segment of $q_{i}^{\text {ed }}, q_{i}^{\mathrm{nl}}$, and $q_{i}^{\text {mix }}$ is presented and compared with the measured SGS flux $q_{i}$ shown in Fig. 6a (same as Fig. 4a). The SGS heat fluxes obtained with the eddy-diffusion model $q_{i}^{\text {ed }}$ (Fig. 6b) show poor correlation (around 35\%) with the measured fluxes (see correlation coefficients in Table 3). Particularly, the eddy-diffusion model underestimates the peak values as well as the overall average (see Table 4) of the three components of the flux. The SGS heat fluxes obtained with the nonlinear model $q_{i}^{\text {nl }}$ (Fig. 6c) show a relatively good correlation (around $80 \%$ ) with the measured fluxes (see Table 3), although the model tends to overestimate the peak values and also the mean and standard deviation (see Table 4). The mixed model provides the most realistic results, although still slightly overestimating the peak values. At this filter scale, the SGS fluxes already represent about $10 \%$ of the streamwise flux, $\left\langle u^{\prime}{ }_{1} \theta^{\prime}\right\rangle$, and about $30 \%$ of the vertical flux, $\left\langle u^{\prime}{ }_{3} \theta^{\prime}\right\rangle$, and thus become important.

Figure 7 presents the PDF of the measured and modeled 

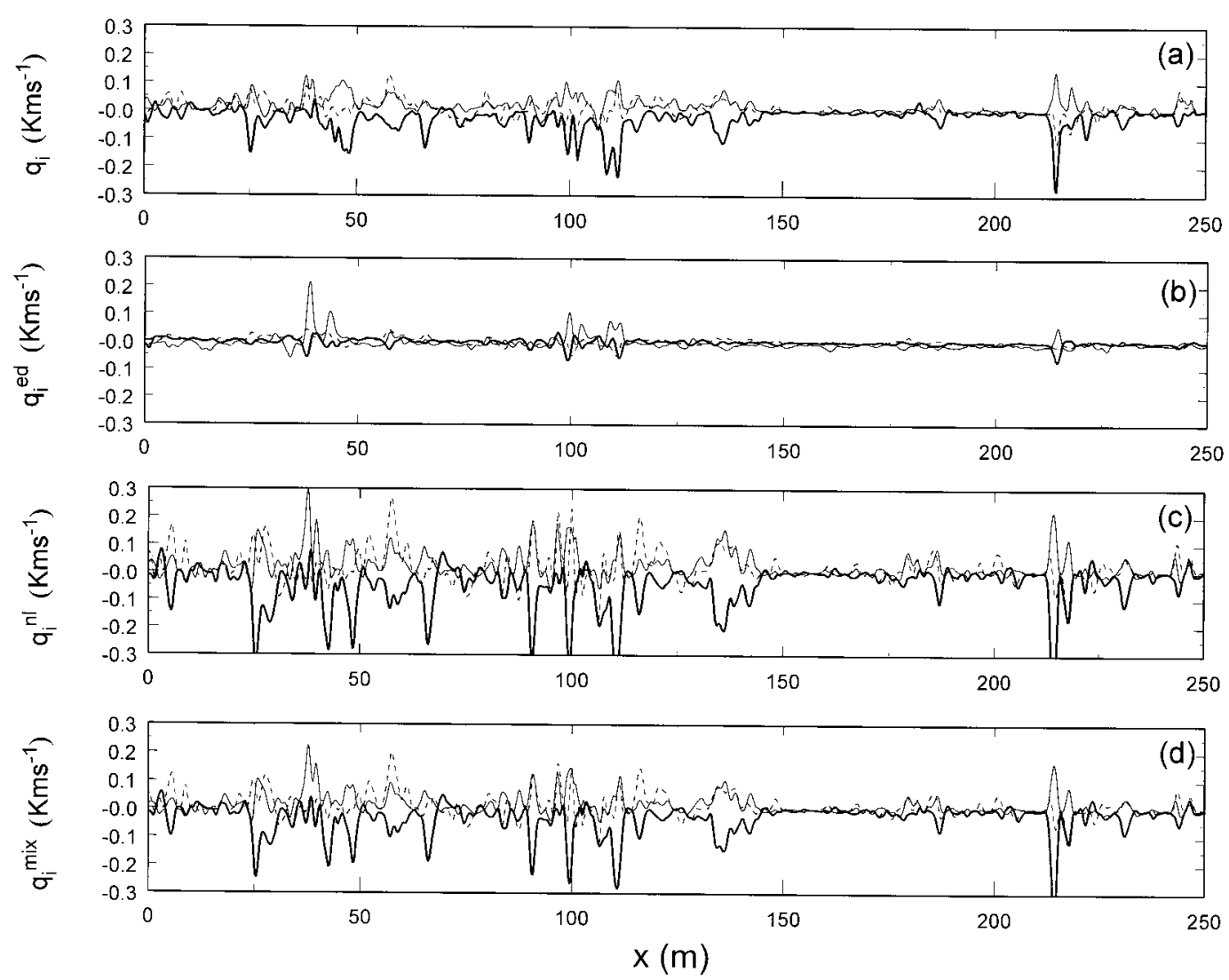

FIG. 6. Portion of the three components of the SGS heat flux signals: streamwise $q_{1}$ (thick solid line), spanwise $q_{2}$ (dashed line), and vertical $q_{3}$ (thin solid line). (a) Measured fluxes, (b) eddy-diffusion model, (c) nonlinear model, and (d) mixed model.

(using the three models) SGS heat fluxes. The PDF of the modeled fluxes, using the nonlinear model (Fig. 7c) and the mixed model (Fig. 7d) show the qualitative asymmetry in the streamwise and vertical components that is found in the measured fluxes (Fig. 7a). The eddy-diffusion model yields SGS fluxes whose PDFs are very different from the measured fluxes (Fig. 7b). $q_{1}^{\text {ed }}$ has a much more symmetric distribution than $q_{1}$, while the PDF of $q_{3}^{\text {ed }}$ is much more asymmetric than the one of $q_{3}$.

The three components of the modeled SGS dissipation are computed with the modeled SGS flux according to their definition $\chi_{m}^{\bmod }=-q_{m}^{\bmod }\left(\partial \tilde{\theta}^{\prime} / \partial x_{i}\right)$, where the superscript mod stands for the modeled quantities (mod can be either ed, nl, or mix). In Figs. 8b-d a segment of the three components of the modeled SGS dissipation of temperature variance $\left(\chi_{1}^{\text {mod }}, \chi_{2}^{\text {mod }}, \chi_{3}^{\text {mod }}\right)$ as well as the total dissipation $\chi^{\text {mod }}=\chi_{1}^{\text {mod }}+\chi_{2}^{\text {mod }}+\chi_{3}^{\text {mod }}$ is presented, obtained using the eddy-diffusion model $\left(\chi^{\mathrm{ed}}\right)$, the nonlinear model $\left(\chi^{\mathrm{nl}}\right)$, and the mixed model $\left(\chi^{\mathrm{mix}}\right)$, respectively. The measured SGS dissipation is plotted in Fig. 8a. As in Fig. 7 for the SGS flux signal, the eddy-diffusion model underestimates the peaks in the SGS dissipation signal. Even though the mean dissipation is correct (recall that the coefficients for the three models are chosen so that the modeled overall dissipation matches the measured dissipation), the standard deviation of the modeled SGS dissipation obtained with the eddy-diffusion model is very small compared with the measurements (see Table 4). The nonlinear model yields a SGS dissipation that is closer to the measured values, although it tends to overestimate the peak values. Again, the mixed model yields the most realistic results, although it still slightly overestimates the peak values.

The PDFs of $\chi^{\mathrm{ed}}, \chi^{\mathrm{nl}}$, and $\chi^{\mathrm{mix}}$, are presented in Figs.

TABLE 3. Correlation (in percentage) between the measured and modeled SGS variables (using the eddy-diffusion, nonlinear, and mixed models) during the measurement period B.

\begin{tabular}{lccccccc}
\hline \hline \multicolumn{1}{c}{ Model } & $q_{1}$ & $q_{2}$ & $q_{3}$ & $\chi_{1}$ & $\chi_{2}$ & $\chi_{3}$ & $\chi=\Sigma_{\chi i}$ \\
\hline Eddy-diffusion & 33.0 & 32.4 & 43.5 & 67.0 & 28.3 & 64.2 & 67.8 \\
Nonlinear & 84.3 & 80.5 & 71.2 & 91.7 & 87.3 & 74.9 & 82.2 \\
Mixed & 84.7 & 81.7 & 74.6 & 92.1 & 88.7 & 80.8 & 85.7 \\
\hline
\end{tabular}


9b-d and compared with the PDF of the measured SGS dissipation $\chi$ (Fig. 9a). As with the SGS fluxes, the modeled dissipation obtained with the nonlinear and mixed models has a PDF similar to the measured dissipation. However, the relative importance of strong negative SGS dissipation (backscatter) is overestimated by the nonlinear term (Fig. 9c). The nearly single-tailed distribution of $\chi^{\text {ed }}$ (Fig. 9b) is a consequence of the formulation of the eddy-diffusion model [Eq. (1.3)]. The fact that $\chi^{\text {ed }}$ has some negative values is due to the presence of a mean vertical gradient in the filtered temperature $\partial ;\langle\tilde{\theta}\rangle / \partial x_{3}$ which is part of $\partial \tilde{\theta} / \partial x_{3}$ but not of $\partial \tilde{\theta}^{\prime} / \partial x_{3}$. This allows for occasional negative values of the product $\left(\partial \tilde{\theta} / \partial x_{3}\right)\left(\partial \tilde{\theta}^{\prime} / \partial x_{3}\right)$, and therefore of the third component of the modeled SGS dissipation, $\chi_{3}^{\text {ed }}$, to occur. The contribution due to mean temperature gradients is of general form $-q_{m}\left(\partial\langle\tilde{\theta}\rangle / \partial x_{m}\right)$. For the case of setup $\mathrm{B}$, the mean contribution of this term is given by $-\left\langle q_{3}\right\rangle \partial\langle\tilde{\theta}\rangle / \partial x_{3}=0.4 \times 10^{-3} \mathrm{~K}^{2} \mathrm{~s}^{-1}$, and represents only $4.8 \%$ of $\langle\chi\rangle$.

\section{c. Effect of filter size}

In this section, data from the four different measurement periods, corresponding to setups $\mathrm{A}, \mathrm{B}, \mathrm{C}$, and $\mathrm{D}$, respectively, are used to study the effect of the filter size on the SGS variables. As shown in Table 1, the meteorological and turbulence conditions during the four measurement periods are very similar. The overall mean SGS heat fluxes and SGS dissipation, computed as in section $3 \mathrm{a}$, are presented in Table 2 for the four setups. An increase in the filter size $\Delta$ is associated with an increase in the magnitude of the mean SGS heat fluxes, due to the larger relative contribution of the subgrid scales to the overall fluxes (some of the increase is also due to slightly different conditions prevalent during the different setups-compare the mean vertical heat fluxes in Table 1). The increase with scale is in agreement with the results from the 1D analysis presented by Porté-Agel et al. (1998). Much smaller changes occur in the mean SGS dissipation when changing the filter size $\Delta$. Recall that the SGS dissipation is independent of scale in an ideal inertial range.

Next, we explore the validity of the assumption of scale invariance of the model coefficients (on which most of the SGS models rely). The model coefficients $\operatorname{Pr}_{T}^{-1} C_{S}^{2}$ (and its components $C_{S}$ and $\operatorname{Pr}_{T}$ ), $C_{\mathrm{nl}},\left[\operatorname{Pr}_{T}^{-1} C_{S}^{2}\right]^{*}$, and $\left[C_{\mathrm{nl}}\right]^{*}$ are computed for the four setups $\mathrm{A}, \mathrm{B}, \mathrm{C}$, and D. In order to obtain an indication of statistical convergence of the results, for each 30-min measurement period, the coefficients are computed for three subperiods of $10 \mathrm{~min}$ each. These values are used to evaluate an approximate standard deviation around the mean coefficient (since only three samples are used, the values computed do not represent a converged standard deviation, but are used here only as a qualitative measure of convergence).

Figures 10a-c show the mean value of the computed 

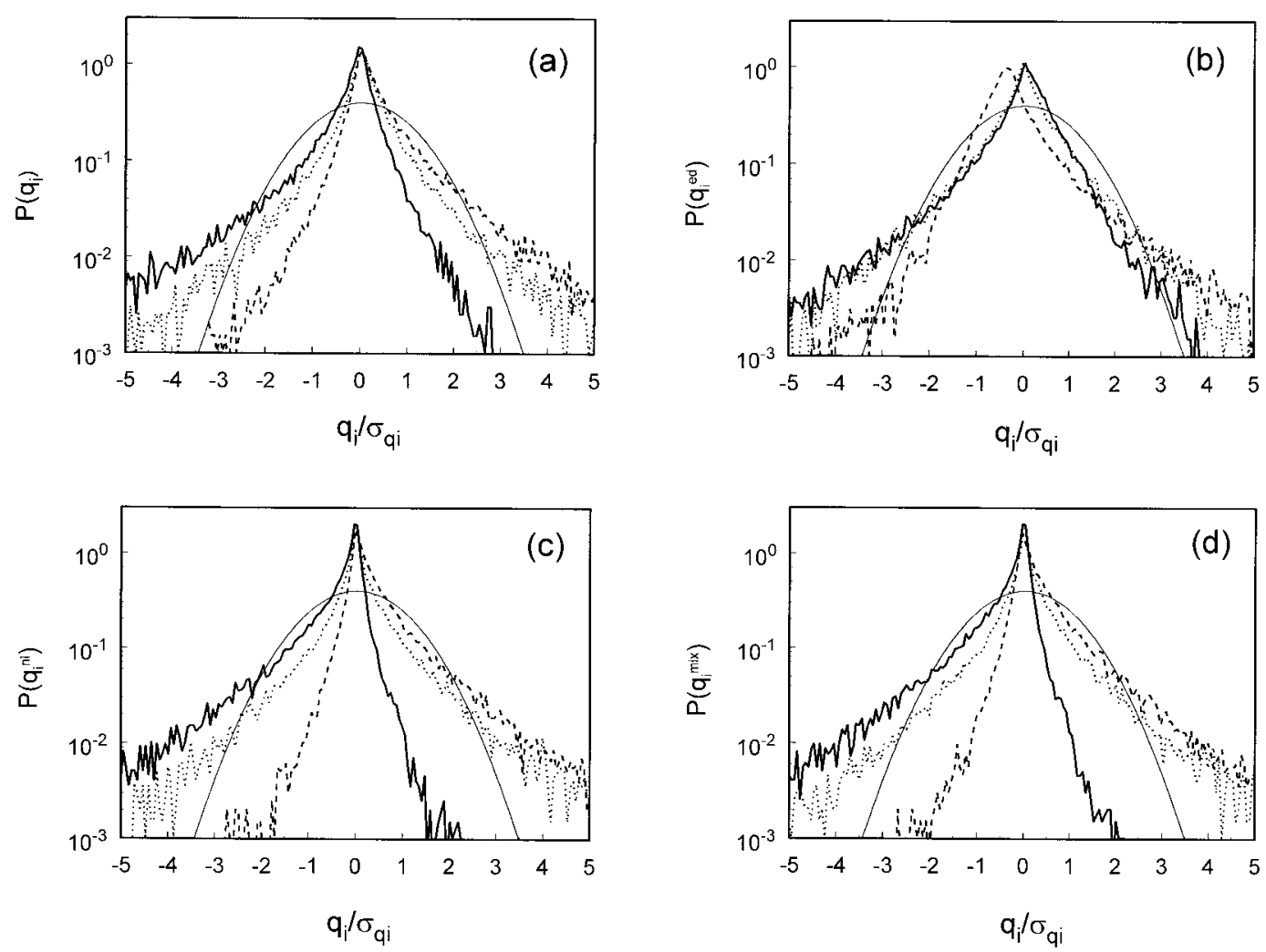

FIG. 7. PDF of the three components of the measured and modeled SGS heat flux, normalized with their standard deviation. Streamwise $q_{1}$ (solid line), spanwise $q_{2}$ (dotted line), and vertical $q_{3}$ (dashed line). The thin solid line is the Gaussian distribution with zero mean. (a) Measured fluxes, (b) eddy-diffusion model, (c) nonlinear model, and (d) mixed model.

model coefficients $\operatorname{Pr}_{T}^{-1} C_{S}^{2}, C_{S}$, and $\operatorname{Pr}_{T}$ as a function of $z / \Delta$ obtained using different streamwise fiters. Note that the change in $z / \Delta$ is only due to changes in $\Delta$ since $z$ is the same for the four setups. From Fig. 10, the streamwise filter has little effect on the main trends of the computed coefficients. The values of $C_{S} \sim 0.1$ and $\operatorname{Pr}_{T} \sim 0.3$ at large $z / \Delta$ (filter size $\Delta$ small relative to the distance to the ground $z$ ) are consistent with previous results [for $C_{S} \sim 0.1$, see Deardorff (1971) and Piomelli et al. (1988); and for $\operatorname{Pr}_{T} \sim 0.4$, see Mason and Derbyshire (1990) and Moin et al. (1991)]. From Figs. 10a and $10 \mathrm{~b}$ there is evidence of scale dependence of the model coefficients for the eddy-diffusion and eddy-viscosity models, $\operatorname{Pr}_{T}^{-1}, C_{S}^{2}$, and $C_{S}$. In the near-ground region, where $z / \Delta$ is small, the filter scale is by necessity on the order of the distance to the ground. For small $z /$ $\Delta(<1)$, the filter operation(s) is performed outside of the inertial subrange of turbulence, and therefore the assumption of scale invariance (based on the existence of an idealized inertial subrange) is violated. The variation of $C_{S}$ with $z / \Delta$ shown in Fig. 10b is in good agreement with the results obtained from simulations (under neutral stability conditions) using the dynamic eddy-viscosity model and presented by Porté-Agel et al. (2000a). These results motivated the authors to propose a new scale-dependent dynamic model that allows the coefficient to change with scale.

The values of $C_{\mathrm{nl}}$ obtained from the four setups under consideration are presented in Fig. 11. Also in this case, the value of $C_{\mathrm{nl}} \sim 0.3$, corresponding to large $z / \Delta$ (filter size $\Delta$ small relative to the distance to the ground $z$ ) is consistent with the values reported in the literature (e.g., Meneveau and Katz 1999a). Again, we find a dependence of the model coefficient $C_{\mathrm{n}}$ on $z / \Delta$. In this case the value of the coefficient increases with an increment of the filter size at the same height.

In Figs. 12a and 12b, the measured values of the coefficients in the mixed model, $\left[\operatorname{Pr}_{T}^{-1} C_{S}^{2}\right]^{*}$ and $\left[C_{\mathrm{nl}}\right]^{*}$, are presented as a function of $z / \Delta$, and compared with the values of $\operatorname{Pr}_{T}^{-1} C_{S}^{2}$ and $C_{\mathrm{nl}}$ corresponding to the eddydiffusion and nonlinear models, respectively. Scale dependence of the two coefficients is also found corresponding to $z / \Delta<1$, although noticeably less than in the case of the pure eddy-diffusion or nonlinear models. Note that the procedure used to compute the mixed model coefficients (see section 3b and the appendix) is based on similarity arguments that assume scale invariance of the coefficients. Hence, the values of the mixed model coefficients obtained using that procedure may not be fully reliable for $z / \Delta<1$. In view of these results, we 

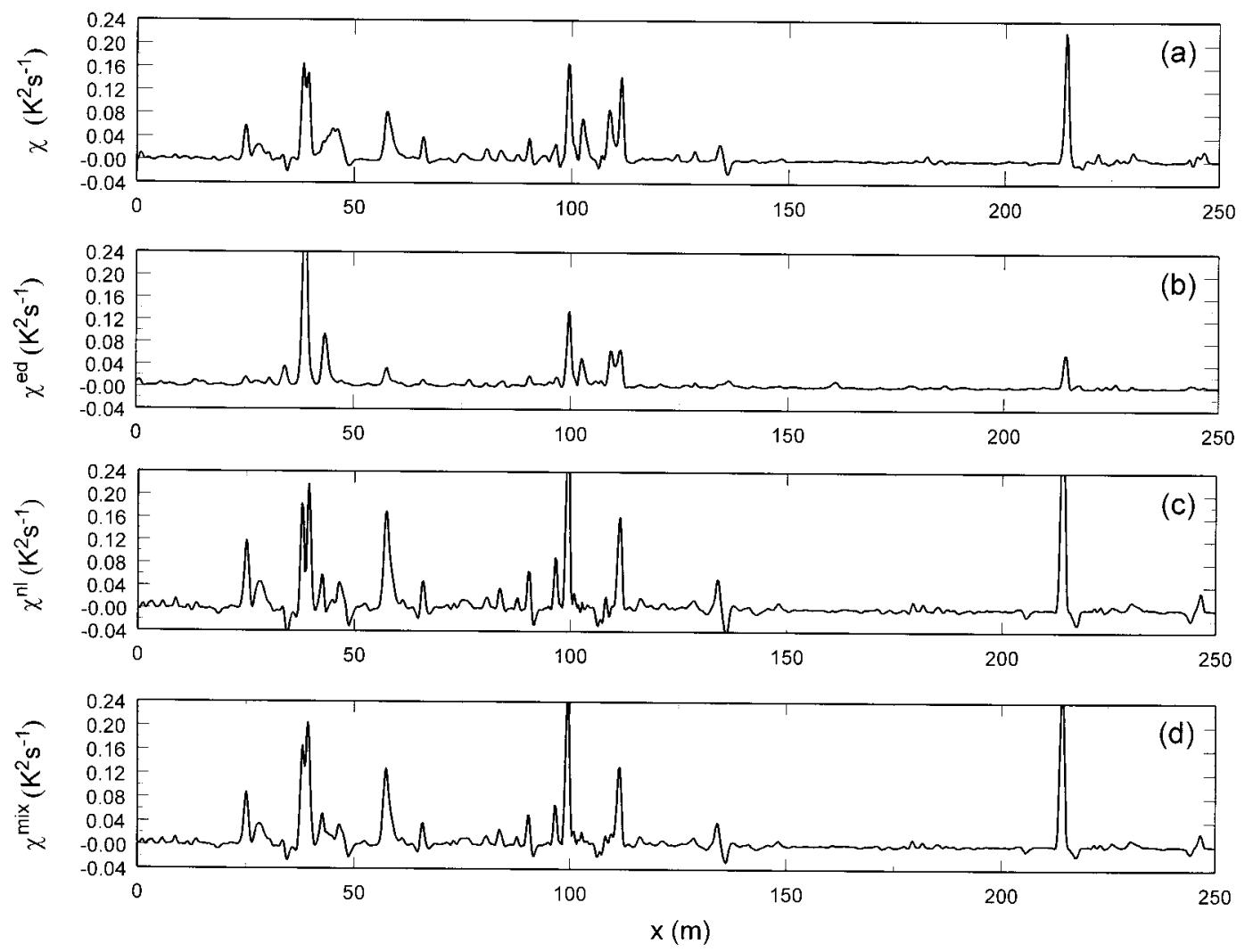

FIG. 8. Portion of the overall SGS dissipation of temperature variance $\chi=\chi_{1}+\chi_{2}+\chi_{3}$. (a) Measured fluxes, (b) eddy-diffusion model, (c) nonlinear model, and (d) mixed model.

believe that the implementation of dynamic mixed models in LES of the ABL (not in practice yet) will benefit from the use of scale-dependent procedures [similar to the scale-dependent dynamic model proposed for the eddy-viscosity model by Porté-Agel et al. (2000a)]. By allowing the model coefficient in the eddy-viscosity model to change with scale, Porté-Agel et al. (2000a) found that simulations of the ABL yield more realistic results.

\section{Conditional averaging and coherent structures}

a. Conditionally averaged SGS variables based on temperature gradients

In order to isolate the relation between coherent structures of the flow (sweeps and ejections) and the SGS dynamics, we perform conditional averaging on the SGS heat fluxes and dissipation of the temperature variance. As in Porté-Agel et al. (2000b), the conditional average of a generic SGS variable $\Phi$ under the condition $C$ in a window of size $X$ is defined according to

$$
\begin{array}{r}
\langle\Phi \mid C\rangle\left(x^{\prime}\right)=\frac{1}{n} \sum_{i=1}^{n} \Phi\left(x_{i}+x^{\prime}, y_{J}, z_{K}\right) \\
-\frac{X}{2} \leq x^{\prime} \leq \frac{X}{2},
\end{array}
$$

where $x_{i}$ (with $1 \leq i \leq n$ ) are the points where $\Phi$ satisfies the condition $C$. As for the SGS fluxes and dissipation, their conditional averages are computed at $y_{J}=0$ and $z_{K}=z_{1}$. Note that for $x^{\prime}=0$ (center of the averaging window) the value of the conditional average $\langle\Phi \mid C\rangle$ is obtained by averaging the value of $\Phi$ at all points that satisfy the condition. Similarly, $\langle\Phi \mid C\rangle$ at any other position $x^{\prime}$ in the averaging window is computed from $\Phi$ at points located at a distance $x^{\prime}$ (upstream if $x^{\prime}<$ 0 and downstream if $x^{\prime}>0$ ) from those satisfying the condition.

Conditional averaging was applied to the SGS heat fluxes $\left(q_{1}, q_{2}\right.$, and $\left.q_{3}\right)$ as well as the three components and the overall SGS dissipation $\left(\chi_{1}, \chi_{2}, \chi_{3}\right.$, and $\left.\chi\right)$. As an attempt to isolate ramp structures in the temperature field, we first use the two conditions employed previously by Porté-Agel et al. (2000b), based on the gradient of the resolved temperature signal.

Condition I: $\partial \tilde{\theta} / \partial t<-\sigma_{\partial \tilde{\theta} / \partial t}$, where $\sigma_{\partial \tilde{\theta} / \partial t}$ is the rms of $\partial \tilde{\theta} / \partial t$. This condition corresponds to a temperature drop in the time series and it tends to highlight the decreasing part of possible ramp structures.

Condition II: $\partial \tilde{\theta} / \partial t>\sigma_{\partial \tilde{\theta} / \partial t}$ corresponds to a temperature increase in the time series. This condition highlights the rising part of the temperature signal.

The selection of the conditioning threshold, $\sigma_{\partial \tilde{\theta} / \partial t}$, is arbitrary and is intended simply to guarantee that it is 

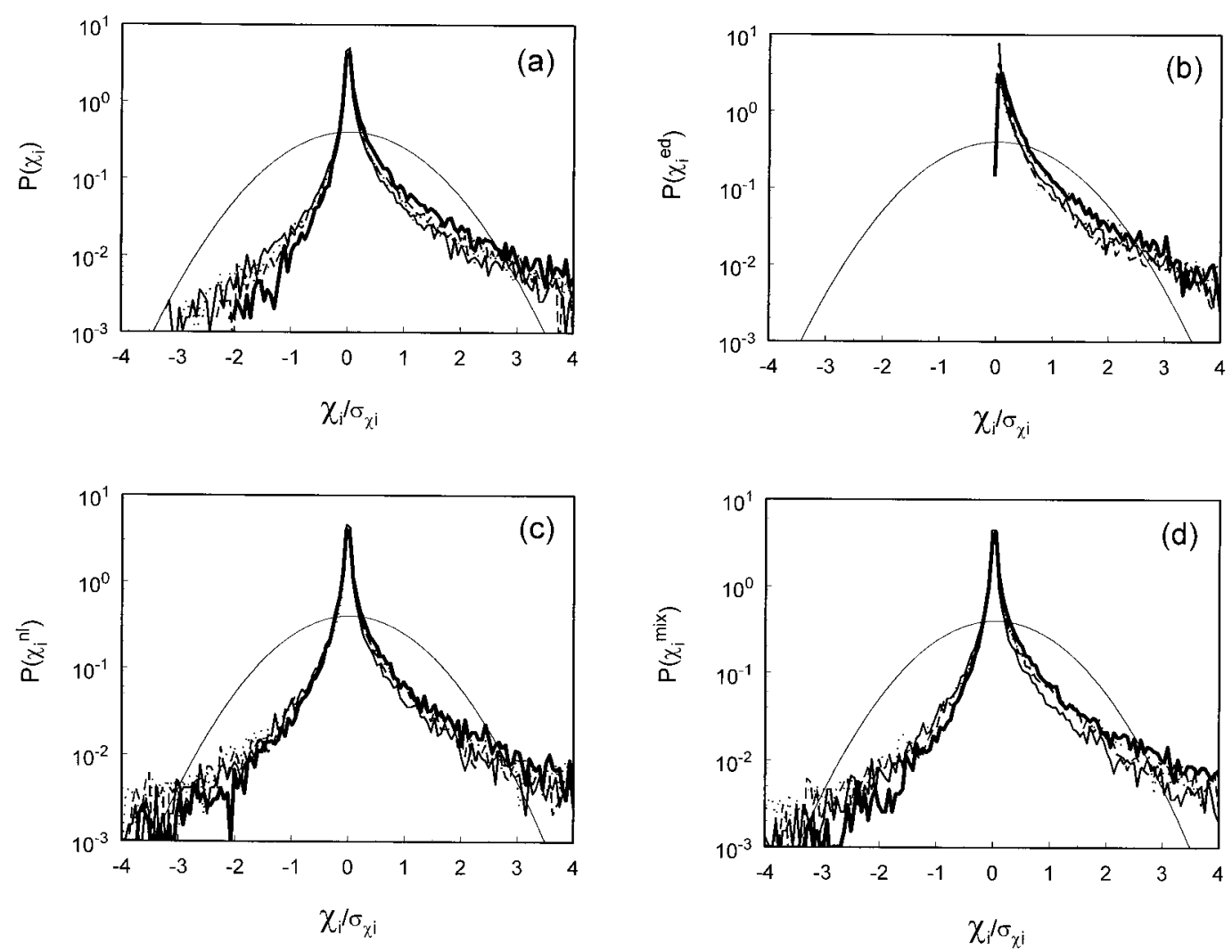

FIG. 9. PDF of the measured and modeled SGS dissipation of temperature variance: streamwise $\chi_{1}$ (solid line), spanwise $\chi_{2}$ (dotted line), vertical $\chi_{3}$ (dashed line), and overall $\chi=\chi_{1}+\chi_{2}+\chi_{3}$ (thick solid line). The thin solid line is the Gaussian distribution with zero mean. (a) Measured fluxes, (b) eddy-diffusion model, (c) nonlinear model, and (d) mixed model.

large enough to isolate the local effects, and small enough to ensure the convergence of conditional averages. The width of the averaging window, $X$, was set to 20 times the filter width, that is, $X=40 \mathrm{~m}$. The conditional average asymptotes to the overall average as we move away from the center of the averaging window (at $\left.x^{\prime}=0\right)$.

The conditional averages for $q_{1}, q_{2}$, and $q_{3}$ under conditions I and II, as well as the overall averages, are presented in Figs. 13a and 13b, respectively. As shown in Table 2, the overall averages are $\left\langle q_{1}\right\rangle=-2.59 \times$ $10^{-2} \mathrm{~K} \mathrm{~m} \mathrm{~s}^{-1},\left\langle q_{2}\right\rangle=+0.30 \times 10^{-2} \mathrm{~K} \mathrm{~m} \mathrm{~s}^{-1}$, and $\left\langle q_{3}\right\rangle=+2.42 \times 10^{-2} \mathrm{~K} \mathrm{~m} \mathrm{~s}^{-1}$. Relatively strong negative temperature gradients, possibly associated with the onset of sweeps of relatively cool air and highlighted by condition I, are associated with SGS fluxes that are larger in magnitude (more than two times larger) than the average. Under condition II (possibly associated with the onset of ejection events), a weaker and more asymmetric change in the SGS fluxes is detected.

The conditional averages for $\chi_{1}, \chi_{2}, \chi_{3}$, and $\chi$ under conditions I and II, as well as the overall averages, are presented in Figs. 14a and 14b, respectively. As shown in Table 2, the overall averages are $\left\langle\chi_{1}\right\rangle=1.90 \times 10^{-3}$ $\mathrm{K}^{2} \mathrm{~s}^{-1},\left\langle\chi_{2}\right\rangle=2.37 \times 10^{-3} \mathrm{~K}^{2} \mathrm{~s}^{-1},\left\langle\chi_{3}\right\rangle=4.05 \times$
$10^{-3} \mathrm{~K}^{2} \mathrm{~s}^{-1}$, and $\langle\chi\rangle=8.33 \times 10^{-3} \mathrm{~K}^{2} \mathrm{~s}^{-1}$. A larger than average (about five times larger) positive value of the streamwise and vertical components of the SGS dissipation, $\chi_{1}$ and $\chi_{3}$, as well as the overall SGS dissipation $\chi$, occurs when there is a strong negative temperature gradient (highlighted by condition I). Thus, under these conditions a relatively large (larger than average) amount of temperature variance is transferred from the large (resolved) to the small (subgrid) scales of the flow. The conditionally averaged spanwise component does not appear to differ substantially from its overall mean. Under condition II, the SGS dissipation $\chi$ is smaller than its mean value. Particularly the streamwise component $\chi_{1}$ is negative and the vertical component $\chi_{3}$ is very small. These results are in qualitative agreement with the results presented by Porté-Agel et al. $(1998,2000 b)$ : for the $1 \mathrm{D}$ and 2D surrogates of the SGS dissipation. However, they also show the important relative contribution of the third component of the SGS dissipation $\chi_{3}$, which highlights the need for using the double array of sensors. In particular, the total dissipation under condition II no longer becomes negative, although it is still significantly reduced. Similar values of the conditional averages are obtained when other fil- 

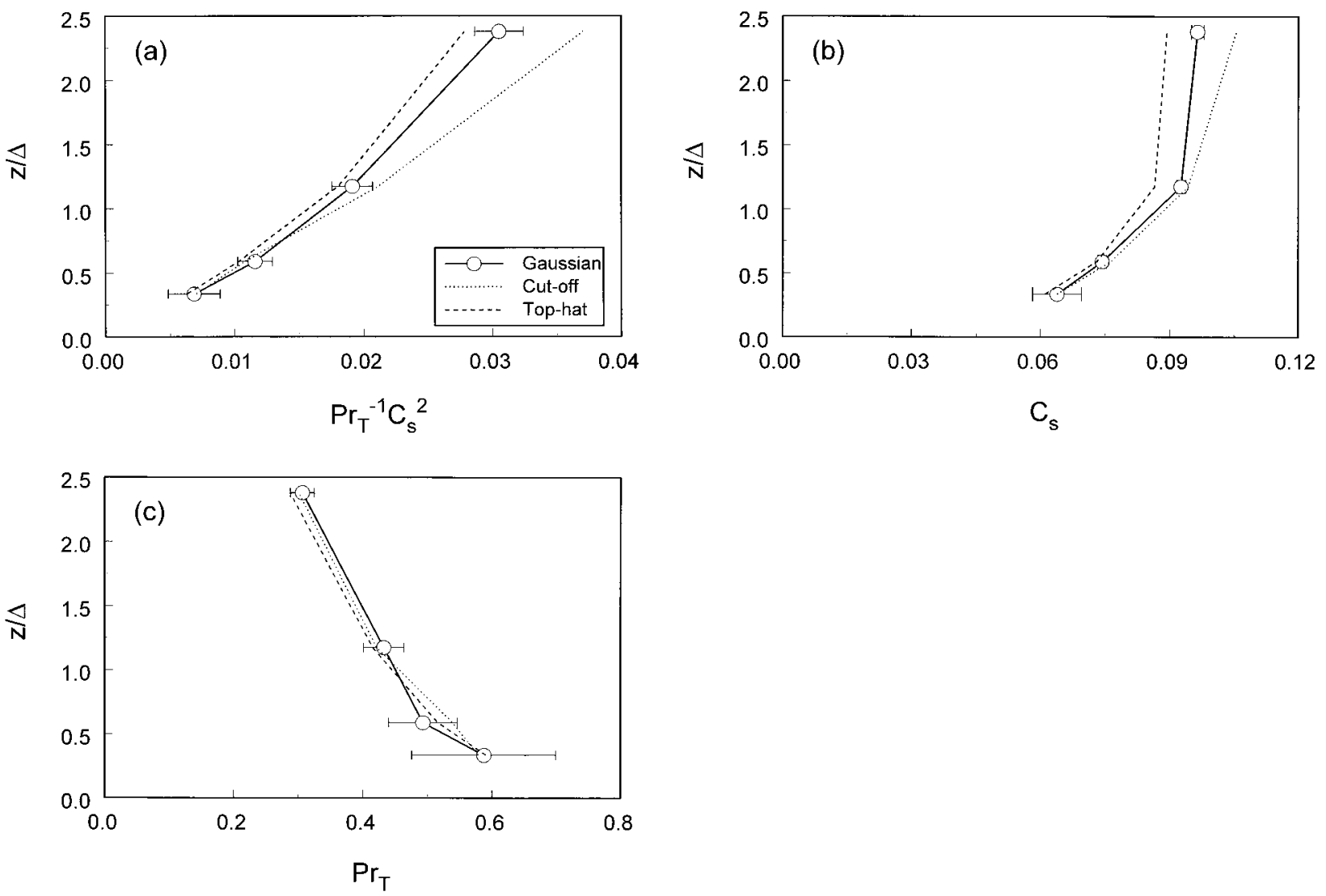

FIG. 10. Value of the model coefficients (a) $\operatorname{Pr}_{T}^{-1} C_{S}^{2}$ in the eddy-diffusion model, and its components: (b) the Smagorinsky coefficient $C_{S}$ and (c) the subgrid-scale Prandtl number $\operatorname{Pr}_{T}$, as a function of $z / \Delta$, obtained for the four setups corresponding to different filter scales $\Delta$ (same height $z$ ). Setup A: $\Delta=1.0 \mathrm{~m}(z / \Delta=2.38)$, setup B: $\Delta=2.0 \mathrm{~m}(z / \Delta=1.18)$, setup C: $\Delta=4.1 \mathrm{~m}(z / \Delta=0.59)$, and setup D: $\Delta$ $=7.1 \mathrm{~m}(z / \Delta=0.33)$. Results are obtained using three different streamwise filters (Gaussian, cutoff, and top-hat). For the Gaussian filter, the open circles are obtained by averaging over three values obtained using 10-min subperiods. The error bars show the approximated standard deviation associated with those three samples.

ters (e.g., spectral cutoff and top-hat) are used instead of the Gaussian filter in the streamwise direction.

Next, we apply conditional averaging using condition I $\left(\partial \tilde{\theta} / \partial t<-\sigma_{\partial \tilde{\theta} / \partial t}\right)$ on the SGS variables obtained from the three models under consideration. Figures $15 \mathrm{~b}-\mathrm{d}$ show the conditionally averaged (using condition I) SGS fluxes modeled with the eddy-diffusion model, nonlinear model, and mixed model, respectively. The conditionally averaged measured SGS fluxes are also shown in Fig. 15a. As mentioned above, the eddy-diffusion model gives SGS heat fluxes whose overall averages are orders of magnitude smaller than the measured flux. The conditionally averaged SGS fluxes are also clearly underestimated by the eddydiffusion model. The nonlinear model overpredicts the SGS fluxes. The mixed model provides a better estimate of both the mean and conditionally averaged SGS fluxes.

Figures 16b-d show the conditionally averaged SGS dissipation (using condition I) modeled with the eddydiffusion model, nonlinear model, and mixed model, respectively. The conditionally averaged measured SGS dissipation is also presented in Fig. 16a. As explained above, the model coefficients were selected so that the mean mod- eled dissipation $\left\langle\chi^{\mathrm{mod}}\right\rangle$ matches the mean measured dissipation $\langle\chi\rangle$. Consistent with the results found for the conditionally averaged SGS fluxes (see Fig. 15), the eddydiffusion model clearly underpredicts the values of $\left\langle\chi_{1} \mid I\right\rangle$, while $\left\langle\chi_{3}^{\text {ed }} \mid I\right\rangle$ shows a much more realistic behavior. The nonlinear and mixed models yield different results: $\left\langle\chi_{3} \mid I\right\rangle$ is very well captured by these two models, while the relative contribution of $\left\langle\chi_{1}^{\bmod } \mid I\right\rangle$ and $\left\langle\chi_{2}^{\bmod } \mid I\right\rangle$ appears to be overestimated (more in the case of the nonlinear model). Similar qualitative behavior is found when condition II $\left(\partial \tilde{\theta} / \partial t>\sigma_{\partial \tilde{\theta} / t}\right)$ is applied to the three models (results not shown): the eddy-diffusion model underestimates the trends in the conditionally averaged SGS dissipation, while the nonlinear and mixed models (particularly the nonlinear model) intensify those trends. Again, the mixed model yields the most realistic results.

\section{b. Conditional velocity and temperature fields based on SGS dissipation}

Next, we use data from the vertical array of anemometers (setup E) to study the vertical distribution 


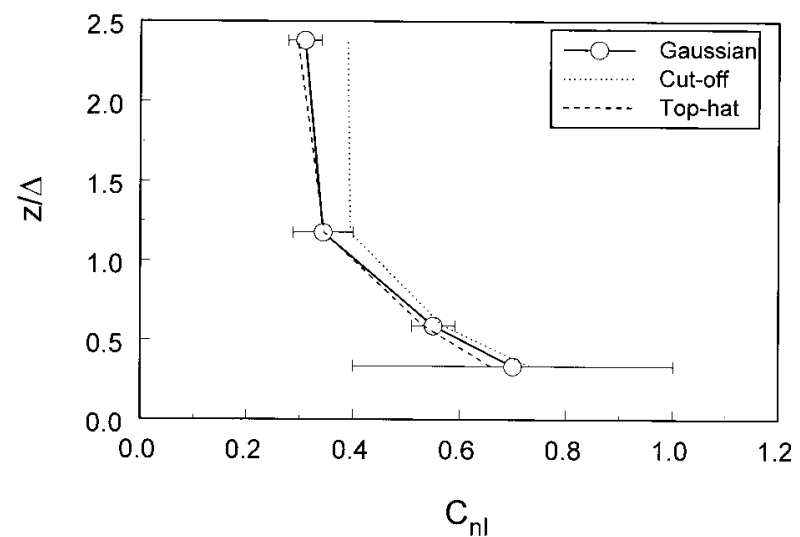

FIG. 11. Value of the model coefficient $C_{\mathrm{nl}}$ in the nonlinear model, as a function of $z / \Delta$, obtained for the four setups corresponding to different filter scales $\Delta$ (same height $z$ ). Setup A: $\Delta=1.0 \mathrm{~m}(z / \Delta=$ $2.38)$, setup B: $\Delta=2.0 \mathrm{~m}(z / \Delta=1.18)$, setup C: $\Delta=4.1 \mathrm{~m}(z / \Delta$ $=0.59)$, and setup D: $\Delta=7.1 \mathrm{~m}(z / \Delta=0.33)$. Results are obtained using three different streamwise filters (Gaussian, cutoff, and tophat). For the Gaussian filter, the open circles are obtained by averaging over three values obtained using 10 -min subperiods. The error bars show the approximated standard deviation associated with those three samples.

of the coherent structures of the flow associated with strong positive and negative SGS dissipation of scalar variance. Instead of using the resolved scales to conditionally average SGS variables, in this section we use the SGS dissipation as criterion, and measure the conditionally averaged resolved velocity and temperature field. The goal is to identify the large-scale conditional flow structure responsible for forward scatter and backscatter events. This approach was used in Piomelli et al. (1996) to analyze DNS data of channel flow at low Reynolds numbers, and by Lin (1999) to analyze LES results.

The filtered variables are computed from the field measurements at each height using a 1D filter that consists of a Gaussian filter in the streamwise direction combined with Taylor's hypothesis. The filter size was $\Delta=2.0 \mathrm{~m}$. Although $1 \mathrm{D}$-filtered variables are expected to be quantitatively different from the corresponding 2D- and 3D-filtered variables, no significant changes between the qualitative behavior of their conditional averages is expected (see Porté-Agel et al. 2000b). The SGS heat flux and a 2D surrogate of the SGS dissipation are obtained at the height of the sixth sensor $z=z_{(6)}=1.78 \mathrm{~m}$ (see section $2 \mathrm{a}$ for a description of setup E). The 2D surrogate of the SGS dissipation is computed from the streamwise and vertical components, that is, $\chi^{2 \mathrm{D}}=-q_{1}\left(\partial \tilde{\theta}^{\prime} / \partial x_{1}\right)-$ $q_{3}\left(\partial \tilde{\theta}^{\prime} / \partial x_{3}\right)$. Both streamwise and vertical derivatives are approximated using centered finite differences, invoking Taylor's hypothesis in the streamwise direction, and using the difference between the values at heights $z_{(7)}$ and $z_{(5)}$ in the vertical direction.

The 2D (streamwise and vertical) filtered velocity and temperature fields are conditionally sampled (a)

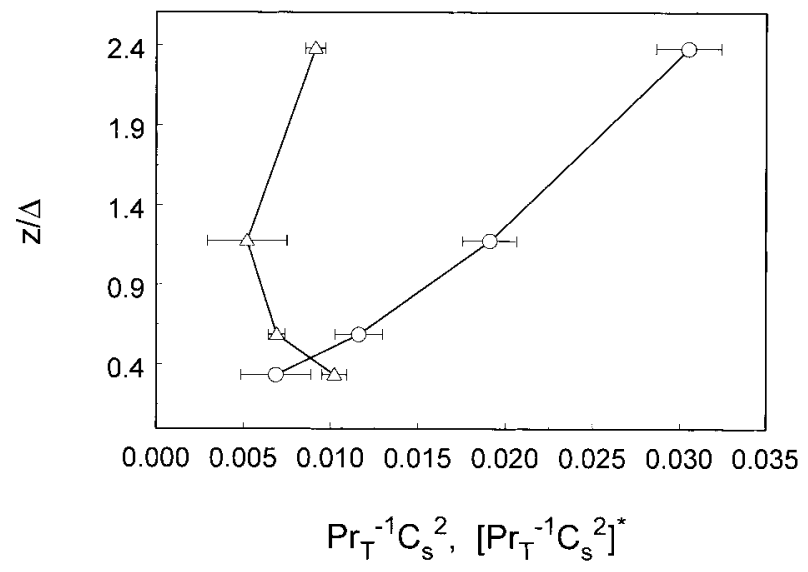

(b)

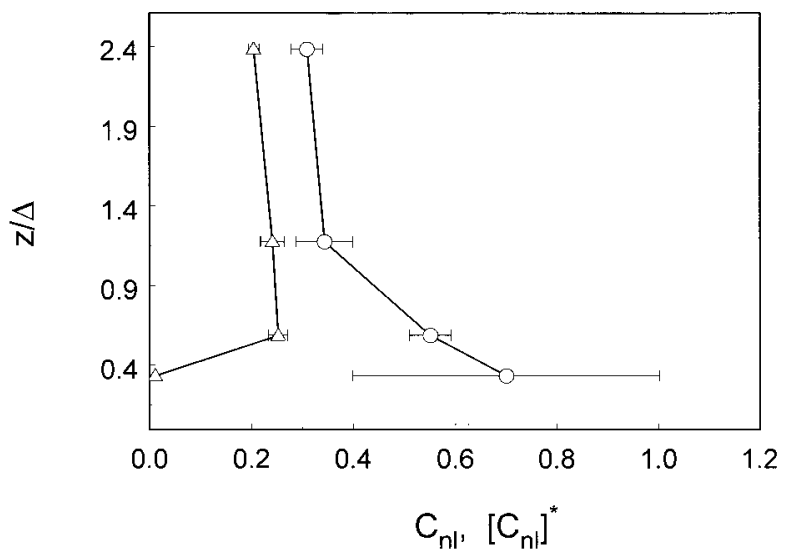

FIG. 12. Value of the model coefficients (a) $\left[\operatorname{Pr}_{T}^{-1} C_{S}^{2}\right]^{*}$ and (b) $\left[C_{\mathrm{nl}}\right]^{*}$ (triangles) in the mixed model, as a function of $z / \Delta$, obtained for the four setups corresponding to different filter scales $\Delta$ (same height $z$ ). The corresponding values of $\operatorname{Pr}_{T}^{-1} C_{S}^{2}$ and $C_{\mathrm{nl}}$ (circles) are also shown (same as in Fig. 10a and Fig. 11, respectively). The triangles and circles are obtained by averaging over three values obtained using 10-min subperiods. The error bars show the approximated standard deviation associated with those three samples.

based on the value of the SGS dissipation. In this case the conditional average of the generic variable $\Phi(\Phi$ $=\tilde{u}_{1}, \tilde{u}_{3}$ or $\left.\tilde{\theta}\right)$ under condition $C$ on a rectangular window of width $\mathrm{X}$ and height $Z\left(=z_{(12)}-z_{(1)}\right)$ is defined as

$$
\begin{aligned}
\langle\Phi \mid C\rangle\left(x^{\prime}, z\right)=\frac{1}{n} \sum_{i=1}^{n} \Phi\left(x_{i}\right. & \left.+x^{\prime}, z\right), \\
-\frac{X}{2} & \leq x^{\prime} \leq+\frac{X}{2} \text { and } \\
z_{(1)} & \leq z \leq z_{(12)},
\end{aligned}
$$

where $x_{i}$ (with $1 \leq i \leq n$ ) is the streamwise coordinate of the points where $\Phi$ satisfies the condition $C$. Two conditions are used based on the value of the $2 \mathrm{D}$ sur- 

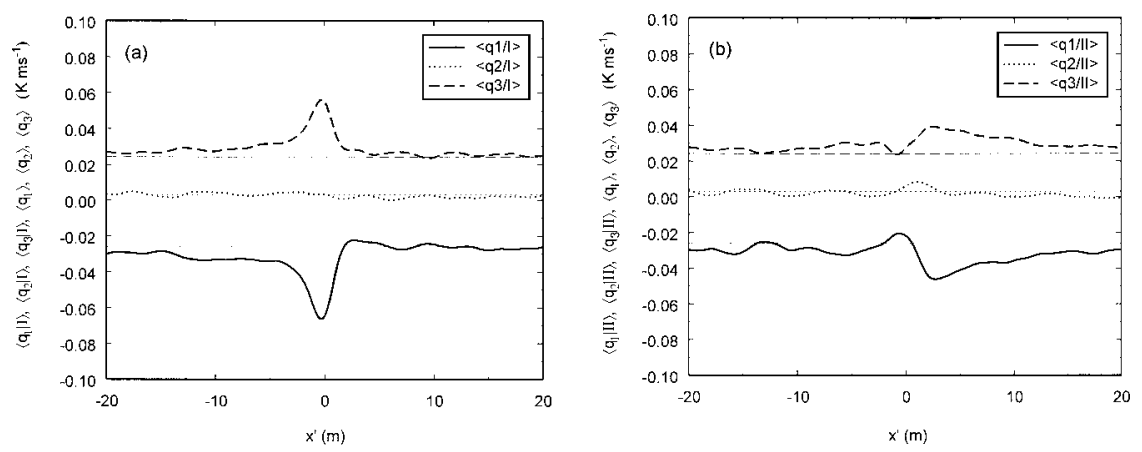

FIG. 13. Conditionally averaged SGS heat fluxes $\left(q_{1}, q_{2}\right.$, and $\left.q_{3}\right)$, under (a) condition I ( $C \equiv$ $\left.\partial \tilde{\theta} / \partial t<-\sigma_{\partial \tilde{\theta} / \partial t}\right)$ and $(\mathrm{b})$ condition II $\left(C \equiv \partial \tilde{\theta} / \partial t>\sigma_{\partial \tilde{\partial} / \partial t}\right)$, for setup B. Light lines correspond to the overall means.

rogate of the SGS dissipation $\left(\chi^{2 \mathrm{D}}\right)$ obtained at $z=$ $z_{(6)}=1.78 \mathrm{~m}$.

Condition $C_{\chi(+)}: \chi^{2 \mathrm{D}}>3\left\langle\chi^{2 \mathrm{D}}\right\rangle$, where $\left\langle\chi^{2 \mathrm{D}}\right\rangle$ is the overall average of the $2 \mathrm{D}$ surrogate of the SGS dissipation. This condition corresponds to large positive values of the SGS dissipation (strong forward scatter).

Condition $C_{\chi(-)}: \chi^{2 \mathrm{D}}<-\left\langle\chi^{2 \mathrm{D}}\right\rangle$. This condition corresponds to relatively large negative values of the SGS dissipation, associated with backscatter of temperature variance.

The thresholds $3\left\langle\chi^{2 \mathrm{D}}\right\rangle$ and $-\left\langle\chi^{2 \mathrm{D}}\right\rangle$ are again selected to guarantee the good convergence of the conditional averaged fields. From here on, the point $x^{\prime}$ $=0$ and $z=1.78 \mathrm{~m}$ is referred as the "reference point."

The resulting fields are presented in Figs. 17a and 17b. Strong forward scatter (positive dissipation) of temperature variance at the reference point is associated with the end part of ejections, clearly identified by the high values of the temperature and vertical velocity downwind $\left(x^{\prime}>0\right)$ of that point (see Fig. 17a). Note also that the reference point is characterized by very strong gradients (drops in time) of the temperature and vertical velocity. This is in good agreement with the results presented in section $4 \mathrm{a}$ (Fig. 14a) that show strong positive dissipation associated with strong drops in the temperature. On the other hand, backscatter (negative SGS dissipation) of temperature variance at the reference point corresponds with the onset of ejections highlighted by the high temperature and vertical velocity found upwind of the that point (Fig. 17b). In this case the gradients in the temperature and vertical velocity (increments in time) are very strong at the reference point, which again is in good agreement with the results presented in section 4a (Fig. 14b), where strong increments (in time) of the temperature are shown to be associated with an important contribution of backscatter.

Next, we perform conditional averaging based on the values of the modeled SGS dissipation $\chi_{\text {mod }}^{2 \mathrm{D}}$ (instead of the measured SGS dissipation $\chi^{2 \mathrm{D}}$ ) in the same fashion as presented above. The modeled SGS fluxes and SGS dissipation, using the eddy-diffusion, nonlinear, and mixed models are computed at the vertical position $z=z_{(6)}$ using 1D filtered variables in Eqs. (1.3), (1.5), and (1.6), respectively. The conditionally averaged fields corresponding to large positive values of the modeled SGS dissipation (condition $C \equiv \chi_{\bmod }^{2 \mathrm{D}}>3\left\langle\chi^{2 \mathrm{D}}\right\rangle$ ) are presented in Figs. $18 \mathrm{~b}-$ $\mathrm{d}$ for the eddy-diffusion, nonlinear, and mixed models. The results are compared with the conditional averages based on large positive values of the measured dissipation and presented in Fig. 18a (equivalent to Fig. 17a). The averaged velocity and temperature profiles are in qualitative agreement with the profiles obtained using the measured
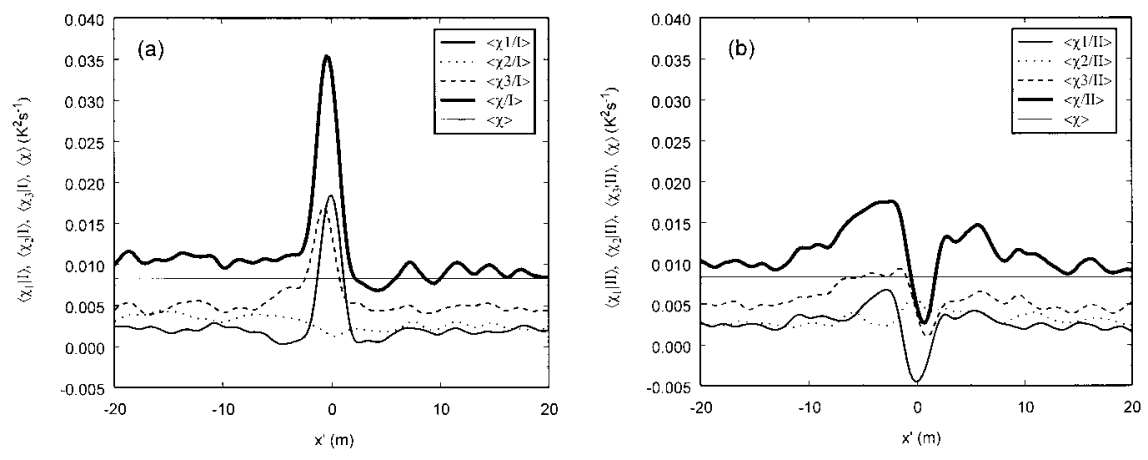

FIG. 14. Conditionally averaged SGS dissipation of temperature variance $\left(\chi_{1}, \chi_{2}, \chi_{3}\right.$ and $\chi=$ $\left.\chi_{1}+\chi_{2}+\chi_{3}\right)$, under (a) condition I $\left(C \equiv \partial \tilde{\theta} / \partial t<-\sigma_{\partial \tilde{\theta} / \partial t}\right)$ and (b) condition II $(C \equiv \partial \tilde{\theta} / \partial t\rangle$ $\left.\sigma_{\partial \tilde{\partial} / \partial t}\right)$, for setup B. The light solid line corresponds to the overall mean SGS dissipation $\langle\chi\rangle$. 

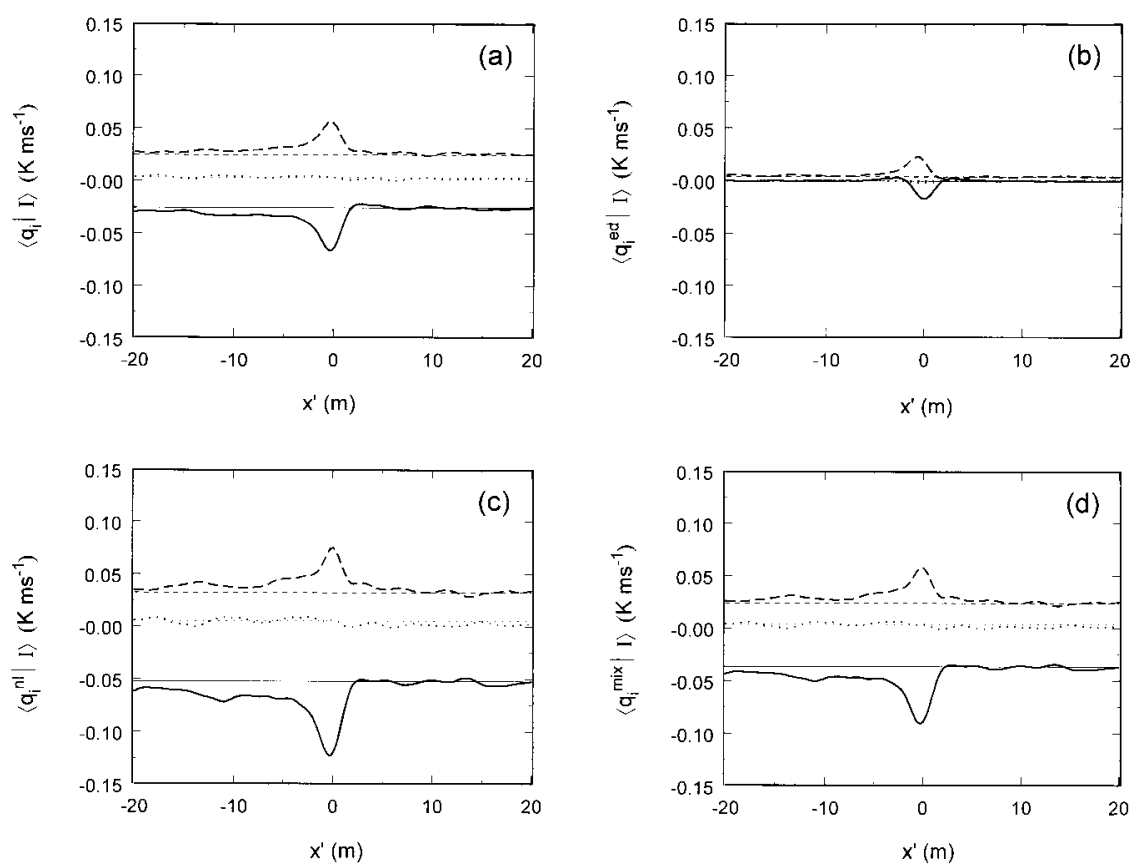

FIG. 15. Conditionally averaged measured and modeled SGS heat flux using condition I ( $C \equiv$ $\left.\partial \tilde{\theta} / \partial t<-\sigma_{\partial \tilde{\theta} / \partial t}\right)$. Streamwise component $\left\langle q_{1} \mid I\right\rangle$ (solid line), spanwise component $\left\langle q_{2} \mid I\right\rangle$ (dotted line), and vertical component $\left\langle q_{3} \mid I\right\rangle$ (dashed line). Light lines correspond to the overall means. (a) Measured fluxes, (b) eddy-diffusion model, (c) nonlinear model, and (d) mixed model.
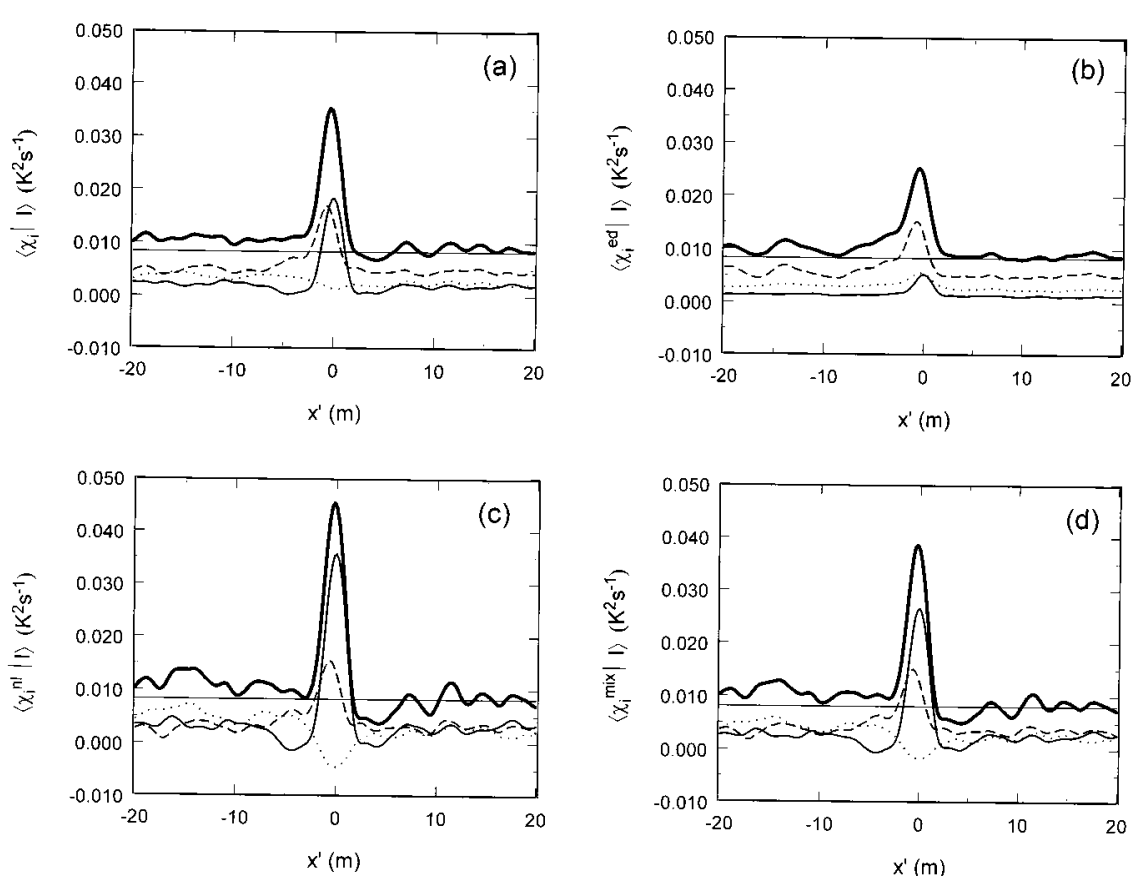

FIG. 16. Conditionally averaged measured and modeled SGS dissipation of temperature variance using condition I $\left(C \equiv \partial \tilde{\theta} / \partial t<-\sigma_{\partial \tilde{\partial} / \partial t}\right)$. Streamwise component $\left\langle\chi_{1} \mid I\right\rangle$ (solid line), spanwise component $\left\langle\chi_{2} \mid I\right\rangle$ (dotted line), vertical component $\left\langle\chi_{3} \mid I\right\rangle$ (dashed line), and overall dissipation $\langle\chi \mid I\rangle$ (thick solid line). The light solid line corresponds to the overall mean SGS dissipation $\langle\chi\rangle$. (a) Measured dissipation, (b) eddy-diffusion model, (c) nonlinear model, and (d) mixed model. 

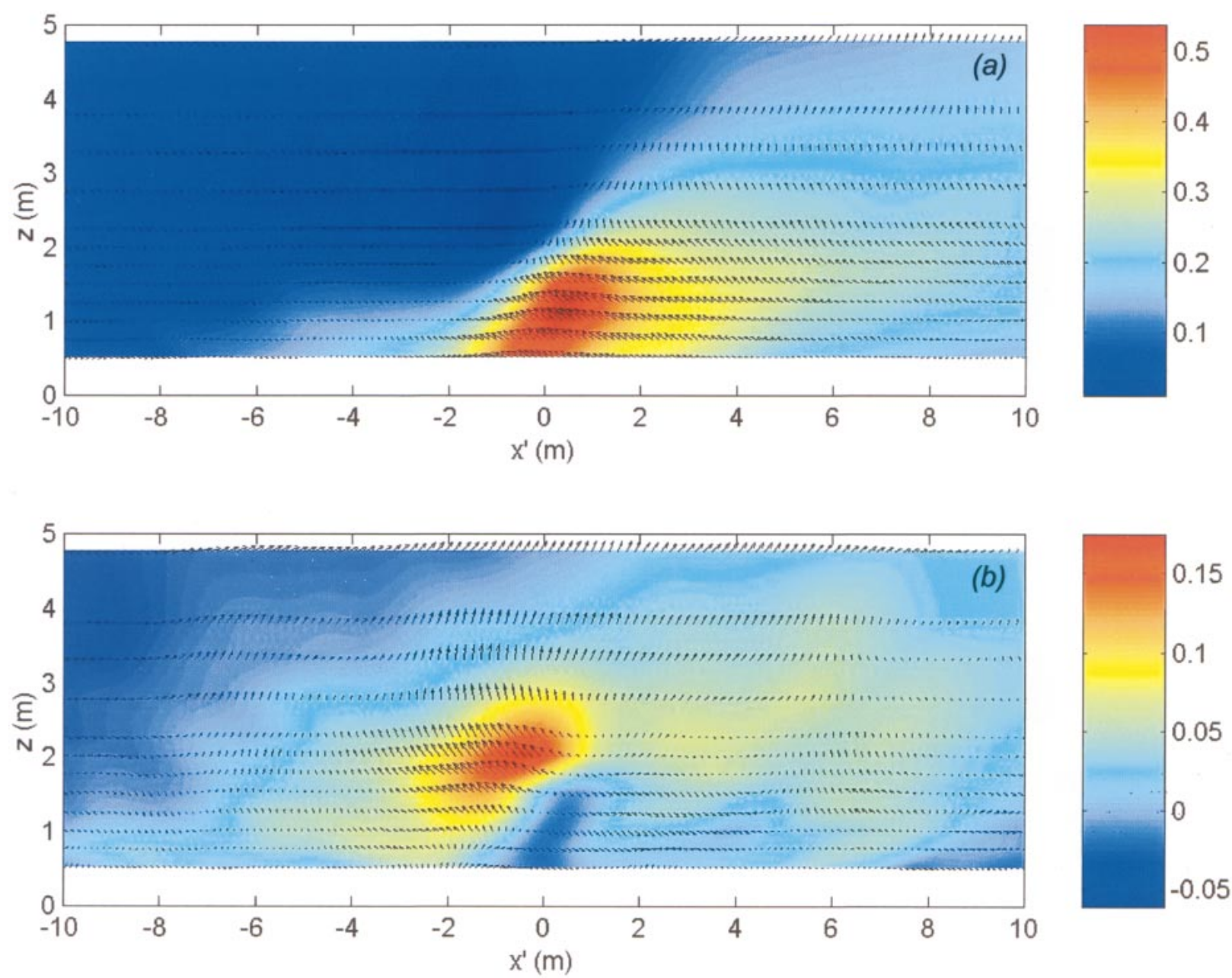

FIG. 17. Conditional averages of the fluctuating part (mean values substracted) of the resolved velocity field (vectors with streamwise and vertical velocity components) and temperature field (color contour plots $-\tilde{\theta}^{\prime}$ in $\mathrm{K}$ ), under (a) condition $C_{\chi(+)}: \chi^{2 \mathrm{D}}>3 \times\left\langle\chi^{2 \mathrm{D}}\right\rangle$ and $(\mathrm{b})$ condition $C_{\chi(-)}: \chi^{2 \mathrm{D}}<-\left\langle\chi^{2 \mathrm{D}}\right\rangle$, for setup $\mathrm{E}$.

real SGS dissipation. Particularly, as explained in section 4a, strong forward scatter (both measured and modeled) occurs during the end part of ejections of relatively warm air, corresponding to strong gradients (drop in time) of temperature and vertical velocity at the reference point $\left(x^{\prime}\right.$ $=0$ and $z=1.78 \mathrm{~m}$ ). However, quantitative differences are found between the magnitude of the conditionally averaged fields obtained in the four cases. In particular, for the case of the eddy-diffusion model, the conditionally sampled temperature appears to be too high during the ejection events (downwind of the reference point). On the other hand, the velocity field corresponding to the eddydiffusion model appears to better match the real velocity field.

The conditionally averaged velocity and temperature fields corresponding to strong backscatter (large negative SGS dissipation) are presented in Figs. 19b and 19c for the nonlinear and mixed models. The results are compared with the corresponding results based on the measured dissipation in Fig. 19a (equivalent to Fig. 17b). Note that no results are presented here for the eddy-diffusion model since the model is fully dissipative, not allowing for negative modeled SGS dissipation. The conditional fields are in good qualitative agreement with the results obtained based on the measured negative SGS dissipation, although some differences are also observed (e.g., higher temperature in the conditionally sampled ejection). Also, high values of the SGS dissipation obtained with the mixed model are associated with the presence of relatively strong sweeps (negative vertical velocity and relatively low temperature) preceding the ejection events (positive vertical velocity and high temperature). This yields particularly strong temperature and vertical velocity gradients at the reference point in the averaging window $\left(x^{\prime}=0\right.$ and $z=$ $1.78 \mathrm{~m})$.

\section{Summary and conclusions}

A field experiment is carried out using 12 3D sonic anemometers placed in the atmospheric surface layer 

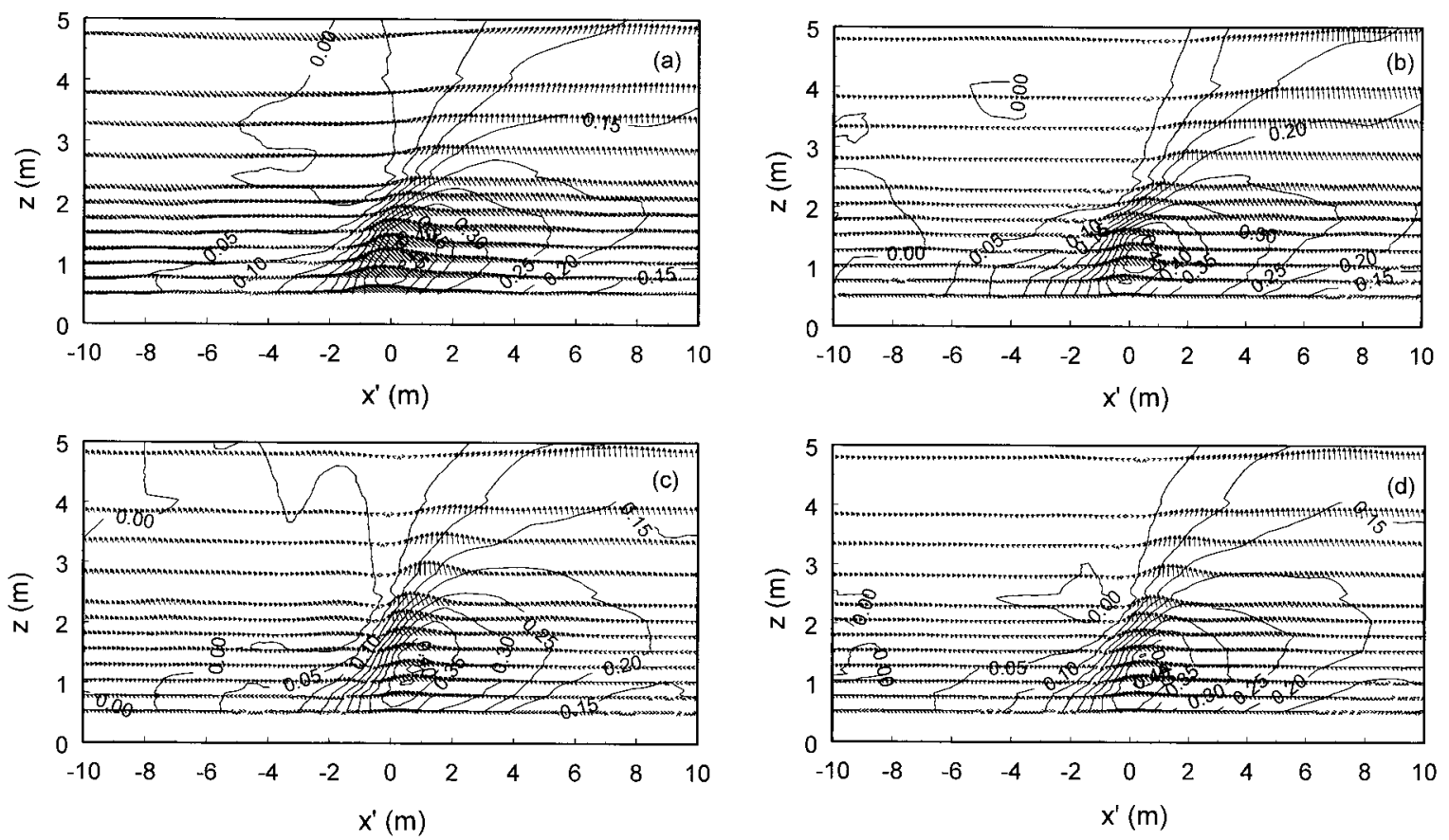

FIG. 18. Conditionally averaged 2D fields of the fluctuating part (mean values substracted) of the resolved velocity field (vectors with streamwise and vertical velocity components) and temperature field (contour plots $-\tilde{\theta}^{\prime}$ in $\mathrm{K}$ ). Conditions are based on the values of (a) the measured SGS dissipation $\left(C_{\chi(+)} \equiv \chi^{2 \mathrm{D}}>3 \times\left\langle\chi^{2 \mathrm{D}}\right\rangle\right)$, (b) the SGS dissipation modeled with the eddy diffusion model $\left(C_{\chi^{(+)}} \equiv \chi_{\mathrm{ed}}^{2 \mathrm{D}}>3 \times\right.$ $\left.\left\langle\chi^{2 \mathrm{D}}\right\rangle\right)$, (c) the nonlinear model $\left(C_{\chi^{(+)}} \equiv \chi_{\mathrm{nl}}^{2 \mathrm{D}}>3 \times\left\langle\chi^{2 \mathrm{D}}\right\rangle\right)$, and (d) the mixed model $\left(C_{\chi^{(+)}} \equiv \chi_{\text {mix }}^{2 \mathrm{D}}>3 \times\left\langle\chi^{2 \mathrm{D}}\right\rangle\right)$.
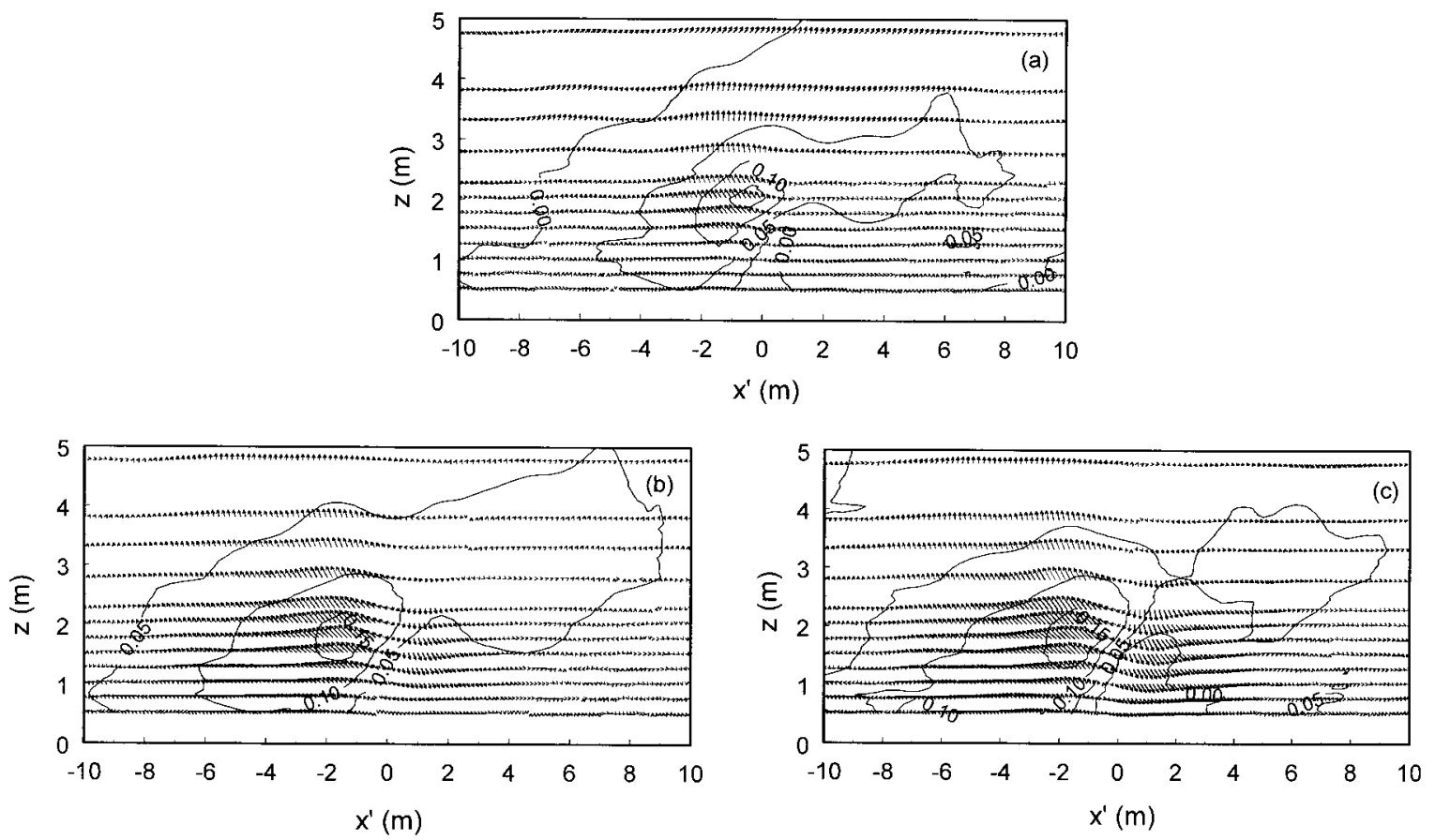

FIG. 19. Conditionally averaged 2D fields of the fluctuating part (mean values substracted) of the resolved velocity field (vectors with streamwise and vertical velocity components) and temperature field (contour plots $-\tilde{\theta}^{\prime}$ in K). Conditions are based on the values of (a) the measured SGS dissipation $\left(C_{\chi^{(-)}} \equiv \chi^{2 D}<-\left\langle\chi^{2 D}\right\rangle\right)$, (b) the SGS dissipation modeled with (b) the nonlinear model $\left(C_{\chi^{(-)}} \equiv \chi_{\mathrm{nl}}^{2 D}<-\left\langle\chi^{2 D}\right\rangle\right)$, and (c) the mixed model $\left(C_{\chi(-)} \equiv \chi_{\text {mix }}^{2 D}<-\left\langle\chi^{2 D}\right\rangle\right)$. 
with the purpose of addressing open issues in SGS modeling for large-eddy simulation. Two major arrangements of the sensors were employed: 1) two horizontal arrays of anemometers placed at two different heights (five anemometers in the upper array and seven in the lower array), and 2) a vertical array. The first arrangement allows us to overcome some of the limitations associated with the 1D and 2D analyses presented by Porté-Agel et al. (1998, 2000b). In particular, since filtered variables (using a 2D filter) can now be computed at two different heights, the vertical gradient of filtered variables is obtained and thus all the contributions (including the vertical) of the SGS dissipation are calculated. We note the relative importance of the vertical contribution to the SGS dissipation, which justifies the use of the double array of sensors.

We evaluate different existing SGS model formulations in terms of their ability to capture the characteristics of the measured SGS variables. Three models are studied: the eddy-diffusion model, the nonlinear (gradient) model, and a mixed model that is a linear combination of the eddy-diffusion model with the nonlinear model. The eddy-diffusion model yields SGS fluxes and dissipation whose statistics (mean values, PDFs) are significantly different from the statistics of the measured SGS variables. For example, the relative contribution of the streamwise and vertical components of the SGS flux are clearly underestimated by the eddy-diffusion model. Furthermore, the eddy-diffusion model is not able to reproduce negative values of the dissipation (backscatter), which are observed in the measurements. SGS variables obtained with the nonlinear and mixed models show higher correlation with the measurements, although the pure nonlinear model significantly overestimates the peak values. While better, the mixed model still slightly overestimates the peak values.

By using different separations between anemometers, we explore the influence of the filter width $\Delta$ on SGS variables. The SGS fluxes increase with increasing filter size, while the mean SGS dissipation does not change substantially with changes in $\Delta$. We also show experimental evidence of the dependence of the model coefficients on the filter scale $\Delta$. This dependence, stronger at smaller $z / \Delta$, is in good agreement with the results obtained from simulations (under neutral stability conditions) using the dynamic eddy-viscosity model and presented by PortéAgel et al. (2000a).

Conditional averaging is used to study the relation of the measured and modeled SGS variables with coherent structures of the flow. This is done by using two types of conditional sampling techniques applied to different variables. As in Porté-Agel et al. (1998, 2000b) conditional averaging is first applied to the measured SGS variables based on the resolved temperature gradients, possibly associated with the onset of sweep and ejection events. Furthermore, conditional averaging is applied to the velocity and temperature fields measured with the vertical array of anemometers, in order to capture the flow structure (projected onto a vertical plane) associated with strong positive and negative SGS dissipation. It is found that under unstable conditions, negative SGS dissipation (backscatter) is most important during the onset of ejections transporting relatively warm air upward. Large positive SGS dissipation of temperature variance is associated with the end of ejections (and/or the onset of sweeps) characterized by strong drops in temperature and vertical velocity under unstable conditions. These results are in qualitative agreement with the 1D and 2D analyses shown by Porté-Agel et al. $(1998,2000 b)$. They also agree qualitatively with the LES results in Lin (1999), who found that the strongest SGS dissipation events (both forward and backward scatter events) occurred during ejections. In considering the motion in the cross-stream plane (not available in our data), he concluded that the backward scatter events occurred near the interface of streamwise vortices and updraft motions. More detailed data analysis is required to identify whether these regions are related to what appears in our streamwise data as the onset of ejection regions.

Conditional averaging is also applied to the modeled SGS variables. Models based on scale-similarity arguments (particularly the mixed model) capture the local dynamics of the subgrid scales (particularly the relative contribution of each component of the SGS dissipation) better than the eddy-diffusion model. However, they tend to overestimate the trends in the conditionally averaged SGS variables. In agreement with Piomelli et al. (1996) and Lin (1999), we conclude that the use of purely stochastic backscatter models cannot reproduce these strong effects of large-scale coherent structures upon SGS dynamics.

Acknowledgments. The authors gratefully acknowledge the dedicated field experimental assistance of their colleagues Markus Pahlow, Elizabeth Jacobs, Pamela Rawe, and Joseph Arreola from The Johns Hopkins University and Zhiming Chen and Ahmed Lachhab from the University of Iowa. The field site coordination was due to the wonderful help of Michael Mata, Roger Shaw, and Susan MacMillan of the University of California, Davis. We also thank Gabriel Katul of Duke University and Jay Ham of Kansas State University for their loan of sonic anemometers and Bert Tanner of Campbell Scientific, Logan, Utah, for discussions and advice on the experimental design and data acquisition system. The authors gratefully acknowledge funding from NSF-ATM-9726270 and USDA-98$35107-6346$.

\section{APPENDIX}

\section{Mixed Models}

The subgrid-scale turbulent flux $q_{i}=\widetilde{u_{i}} \theta-\hat{\tilde{u}}_{i} \hat{\tilde{\theta}}$ can be modeled at the grid scale $\tilde{\Delta}$, using the mixed model, as 


$$
q_{i}^{\text {mix }}=-\left[\operatorname{Pr}_{T}^{-1} C_{S}^{2}\right]^{*} \Delta^{2}|\tilde{S}| \frac{\partial \tilde{\theta}}{\partial x_{i}}+\left[C_{\mathrm{nl}}\right]^{*} \Delta^{2} \frac{\partial \tilde{u}_{i}}{\partial x_{k}} \frac{\partial \tilde{\theta}}{\partial x_{k}} .
$$

If the mixed model is used at a larger scale $\hat{\Delta}=\gamma \tilde{\Delta}$ (with $\gamma>1$ ) and scale invariance of the model coefficients is assumed the SGS flux at that scale, $Q_{i}=$ $\widehat{\vec{u}_{i} \theta}-\hat{\tilde{u}_{i}} \hat{\tilde{\theta}}$, is modeled according to

$Q_{i}^{\operatorname{mix}}=-\left[\operatorname{Pr}_{T}^{-1} C_{S}^{2}\right]^{*}(\gamma \Delta)^{2}|\hat{\tilde{S}}| \frac{\partial \hat{\tilde{\theta}}}{\partial x_{i}}+\left[C_{\mathrm{n}}\right]^{*}(\gamma \Delta)^{2} \frac{\partial \hat{\tilde{\tilde{u}}}}{\partial x_{k}} \frac{\partial \hat{\tilde{\theta}}}{\partial x_{k}}$

We can define the flux

$$
L_{i}=Q_{i}^{\bmod }-\widehat{q_{i}^{\text {mod }}}=\widehat{\tilde{u}_{i}} \widetilde{\tilde{\theta}}-\hat{\tilde{u}}_{i} \hat{\tilde{\theta}} .
$$

Replacing (A1) and (A2) into (A3) yields the identity

$$
L_{i}=\left[\operatorname{Pr}_{T}^{-1} C_{S}^{2}\right] * M_{i}+\left[C_{\mathrm{nl}}\right] * N_{i}
$$

where

$$
\begin{aligned}
& M_{i}=\Delta^{2}\left(\left|\widehat{\bar{S} \mid \frac{\partial \tilde{\theta}}{\partial x_{i}}}-\gamma^{2}\right| \hat{\tilde{S}} \mid \frac{\partial \hat{\tilde{\theta}}}{\partial x_{i}}\right), \quad \text { and } \\
& N_{i}=\Delta^{2}\left(\gamma^{2} \frac{\partial \hat{\tilde{u}}_{i}}{\partial x_{k}} \frac{\partial \hat{\tilde{\theta}}}{\partial x_{k}}-\frac{\partial \tilde{u}_{i} \partial \tilde{\theta}}{\partial x_{k}} \frac{\partial x_{k}}{\partial x_{k}}\right)
\end{aligned}
$$

The error associated with the use of the SGS model in the identity (A4) is

$$
e_{i}=L_{i}-\left(\left[\operatorname{Pr}_{T}^{-1} C_{S}^{2}\right] * M_{i}+\left[C_{\mathrm{n}}\right] * N_{i}\right) .
$$

The square of the error $e_{i}$ averaged over some domain, $E=\left\langle e_{i}^{2}\right\rangle$ can be minimized with espect to $\left[C_{\mathrm{n} 1}\right]^{*}$ by setting

$$
\frac{\partial\left\langle e_{i}^{2}\right\rangle}{\partial\left[C_{\mathrm{nl}}\right]^{*}}=0,
$$

which yields the equation

$$
\left[\operatorname{Pr}_{T}^{-1} C_{S}^{2}\right] *\left\langle M_{i} N_{i}\right\rangle+\left[C_{\mathrm{nl}}\right] *\left\langle N_{i} N_{i}\right\rangle-\left\langle L_{i} N_{i}\right\rangle=0 .
$$

Also, if we want the mean modeled SGS dissipation to match the mean real SGS dissipation (i.e., $\left\langle\chi^{\bmod }\right\rangle=$ $\langle\chi\rangle)$, we can write

$$
\begin{aligned}
\left\langle-q_{i} \frac{\partial \tilde{\theta}^{\prime}}{\partial x_{i}}\right\rangle= & +\left[\operatorname{Pr}_{T}^{-1} C_{S}^{2}\right] *\left\langle\Delta^{2}|\tilde{S}| \frac{\partial \tilde{\theta}}{\partial x_{i}} \frac{\partial \tilde{\theta}^{\prime}}{\partial x_{i}}\right\rangle \\
& -\left[C_{\mathrm{n} 1}\right] *\left\langle\Delta^{2} \frac{\partial \tilde{u}_{i}}{\partial x_{k}} \frac{\partial \tilde{\theta}}{\partial x_{k}} \frac{\partial \tilde{\theta}^{\prime}}{\partial x_{i}}\right\rangle .
\end{aligned}
$$

Thus, we have a system of two equations, (A9) and (A10), and two unknowns, $\left[\operatorname{Pr}_{T}^{-1} C_{S}^{2}\right]^{*}$ and $\left[C_{\mathrm{nl}}\right]^{*}$. Solving this system yields

$$
\begin{aligned}
{\left[C_{\mathrm{n} 1}\right]^{*}=} & \frac{\left\langle M_{i} N_{i}\right\rangle\left\langle q_{i} \frac{\partial \tilde{\theta}^{\prime}}{\partial x_{i}}\right\rangle+\left\langle L_{i} N_{i}\right\rangle\left\langle\Delta^{2}|\tilde{S}| \frac{\partial \tilde{\theta}}{\partial x_{i}} \frac{\partial \tilde{\theta}^{\prime}}{\partial x_{i}}\right\rangle}{\left\langle N_{i} N_{i}\right\rangle\left\langle\Delta^{2}|\tilde{S}| \frac{\partial \tilde{\theta}}{\partial x_{i}} \frac{\partial \tilde{\theta}_{i}^{\prime}}{\partial x_{i}}\right\rangle+\left\langle M_{i} N_{i}\right\rangle\left\langle\Delta^{2} \frac{\partial \tilde{u}_{i}}{\partial x_{k}} \frac{\partial \tilde{\theta}}{\partial x_{k}} \frac{\partial \tilde{\theta}^{\prime}}{\partial x_{i}}\right\rangle}, \text { and } \\
{\left[\operatorname{Pr}_{T}^{-1} C_{S}^{2}\right]^{*}=} & \frac{\left[C_{\mathrm{nl}}\right]^{*}\left\langle\Delta^{2} \frac{\partial \tilde{u}_{i}}{\partial x_{k}} \frac{\partial \tilde{\theta}}{\partial x_{k}} \frac{\partial \tilde{\theta}^{\prime}}{\partial x_{i}}\right\rangle-\left\langle q_{i} \frac{\partial \tilde{\theta}^{\prime}}{\partial x_{i}}\right\rangle}{\left\langle\Delta^{2}|\tilde{S}| \frac{\partial \tilde{\theta}}{\partial x_{i}} \frac{\partial \tilde{\theta}^{\prime}}{\partial x_{i}}\right\rangle} .
\end{aligned}
$$

\section{REFERENCES}

Akselvoll, K., and P. Moin, 1996: Large-eddy simulation of turbulent confined coannular jets. J. Fluid Mech., 315, 387-411.

Albertson, J. D., and M. B. Parlange, 1999a: Natural integration of scalar fluxes from complex terrain. Adv. Water Res., 23, 239-252.

— plications for land-atmosphere interaction over complex terrain Water Resour. Res., 35, 2121-2132.

Anderson, R., and C. Meneveau, 1999: Effects of the similarity model in finite-difference LES of isotropic turbulence using a Lagrangian dynamic mixed model. Flow Turbul. Combust, 62, 201-225.

Andrén, A., A. R. Brown, J. Graf, P. J. Mason, C.-H. Moeng, F. T. M. Nieuwstadt, and U. Schumann, 1994: Large-eddy simulation of the neutrally stratified boundary layer: A comparison of four computer codes. Quart. J. Roy. Meteor. Soc., 120, 1457-1484.

Avissar, R., and T. Schmidt, 1998: An evaluation of the scale at which ground-surface heat flux patchiness affects the convective boundary layer using large-eddy simulations. J. Atmos. Sci., 55, $2666-2689$.

Bardina, J., J. H. Ferziger, and W. C. Reynolds, 1980: Improved subgrid scale models for large-eddy simulation. Amer. Inst. Aeronaut. Astronaut. Paper 80-1357.

Bastiaans, R. J. M., C. C. M. Rindt, and A. A. Van Steenhoven, 1998 Experimental analysis of a confined transitional plume with respect to subgrid-scale modelling. Int. J. Heat Mass Transfer, 41, 3989-4007.

Cerutti, S., and C. Meneveau, 2000: Statistics of filtered velocity in grid and wake turbulence. Phys. Fluids, 12, 1143-1165.

Clark, R. G., J. H. Ferziger, and W. C. Reynolds, 1979: Evaluation of subgrid models using an accurately simulated turbulent flow. J. Fluid Mech., 91, 1-16.

Deardorff, J. W., 1971: On the magnitude of the subgrid-scale eddy coefficient. J. Comput. Phys., 7, 120-33. 
Domaradski, J. A., W. Liu, and M. E. Brachet, 1993: An analysis of subgrid-scale interactions in numerically simulated isotropic turbulence. Phys. Fluids, A5, 1747-1759.

Dwyer, M. J., E. G. Patton, and R. H. Shaw, 1997: Turbulent kinetic energy budgets from a large-eddy simulation of airflow above and within a forest canopy. Bound.-Layer Meteor., 84, 23-43.

Gao, W., and R. H. Shaw, 1992: Conditional analysis of temperature and humidity microfronts and ejection/sweep motions within and above a deciduous forest. Bound.-Layer Meteor., 59, 35-57.

,$- \ldots$, and K. T. Paw U, 1989: Observation of organized structure in turbulent flow within and above a forest canopy. Bound.Layer Meteor., 47, 349-377.

Germano, M., U. Piomelli, P. Moin, and W. H. Cabot, 1991: A dynamic subgrid-scale eddy viscosity model. Phys. Fluids, 43, 1760-1765.

Ghosal, S., T. S. Lund, P. Moin, and K. Akselvoll, 1995: A dynamic localization model for large eddy simulation of turbulent flows. J. Fluid Mech., 286, 229-255.

Härtel, C., L. Kleiser, F. Unger, and R. Friedrich, 1994: Subgrid-scale energy transfer in the near-wall region of turbulent flows. Phys. Fluids, 6, 3130-3143.

Katul, G., C.-I. Hsieh, and J. Sigmon, 1997a: Energy-inertial scale interactions for velocity and temperature in the unstable atmospheric surface layer. Bound.-Layer Meteor., 82, 49-80.

_, G. Kuhn, J. Schieldge, and C.-I. Hsieh, 1997b: The ejectionsweep character of scalar fluxes in the unstable surface layer. Bound.-Layer Meteor., 83, 1-26.

Khanna, S., and J. G. Brasseur, 1998: Three-dimensional buoyancyand shear-induced local structure of the atmospheric boundary layer. J. Atmos. Sci., 55, 710-743.

Kiely, G., J. D. Albertson, M. B. Parlange, and W. E. Eichinger, 1996: Convective scaling of the average dissipation rate of temperature variance in the atmospheric surface layer. Bound.-Layer Meteor., 77, 267-284.

Kosovic, B., 1997: Subgrid-scale modelling for the large-eddy simulation of high-Reynolds-number boundary layers. J. Fluid Mech., 336, 151-182.

Leonard, A., 1974: Energy cascade in large-eddy simulation of turbulent fluid flows. Advances in Geophysics, Vol. 18, Academic Press, 237-248.

Lilly, D. K., 1967: The representation of small-scale turbulence in numerical simulation experiments. Proc. IBM Scientific Computing Symp. Environ. Sci., 195-210.

Lin, C.-L., 1999: Near-grid-scale energy transfer and coherent structures in the convective planetary boundary layer. Phys. Fluids, 11, 3482-3494.

Liu, S., C. Meneveau, and J. Katz, 1994: On the properties of similarity subgrid-scale models as deduced from measurements in a turbulent jet. J. Fluid Mech., 275, 83-119.

,$- \ldots$, and _ _ 1995: Experimental study of similarity subgridscale models of turbulence in the far-field of a jet. Appl. Sci. Res., 54, 177-190.

Mason, P. J., 1989: Large-eddy simulation of the convective atmospheric boundary layer. J. Atmos. Sci., 46, 1492-1516.

_ 1994 : Large-eddy simulation: A critical review of the technique. Quart. J. Roy. Meteor. Soc., 120, 1-26.

— bly-stratified atmospheric boundary layer. Bound.-Layer Meteor., 53, 117-162.

— simulations of boundary layers. J. Fluid Mech., 242, 51-78.

Meneveau, C., 1994: Statistics of turbulence subgrid-scale stresses: Necessary conditions and experimental tests. Phys. Fluids, 6, 815-833.

_ based on the Germano identity. Phys. Fluids, 11, 245-247.

- and — 1999b: Conditional subgrid force and dissipation in locally isotropic and rapidly strained turbulence. Phys. Fluids, 11, 2317-2329.
- , and - 2000: Scale-invariance and turbulence models for large-eddy simulation. Annu. Rev. Fluid Mech., 32, 1-32.

Moeng, C.-H., 1984: A large-eddy-simulation model for the study of planetary boundary-layer turbulence. J. Atmos. Sci., 41, 20522062.

Moin, P., K. D. Squires, and S. Lee, 1991: A dynamic subgrid-scale model for compressible turbulence and scalar transport. Phys. Fluids, A3, 2746-2757.

Nieuwstadt, F. T. M., P. J. Mason, C.-H. Moeng, and U. Schumann, 1991: Large-eddy simulation of the convective boundary layer: A comparison of four computer codes. Turbul. Shear Flows, 8, 343-367.

O'Neil, J., and C. Meneveau, 1997: Subgrid-scale stresses and their modeling in a turbulent plane wake. J. Fluid Mech., 349, 253293.

Parlange, M. B., W. E. Eichinger, and J. D. Albertson, 1995: Regional scale evaporation and the atmospheric boundary layer. Rev. Geophys., 33, 99-124.

Patton, E. G., R. H. Shaw, M. J. Judd, and M. R. Raupach, 1998: Large-eddy simulation of windbreak flow. Bound.-Layer Meteor., 87, 275-306.

Peltier, L. J., J. C. Wyngaard, S. Khanna, and J. G. Brasseur, 1996: Spectra in the unstable surface layer. J. Atmos. Sci., 53, 49-61.

Piomelli, U., P. Moin, and J. H. Ferziger, 1988: Model consistency in large eddy simulation of turbulent channel flows. Phys. Fluids, 31, 1884-1891.

__ , Y. Yu, and R. H. Adrian, 1996: Subgrid-scale energy transfer and near-wall turbulence structure. Phys. Fluids, 8, 215-224

Pope, S. B., 2000: Turbulent Flows. Cambridge University Press, 770 pp.

Porté-Agel, F., C. Meneveau, and M. B. Parlange, 1998: Some basic properties of the surrogate subgrid-scale heat flux in the atmospheric boundary layer. Bound.-Layer Meteor., 88, 425-444.

__ C. Meneveau, and M. B. Parlange, 2000a: A scale-dependent dynamic model for large-eddy simulation: Application to a neutral atmospheric boundary layer. J. Fluid Mech., 415, 261-284.

_ _ M. B. Parlange, C. Meneveau, W. E. Eichinger, and M. Pahlow, 2000b: Subgrid-scale dissipation in the atmospheric surface layer: Effects of stability and filter dimension. J. Hydrometeor., 1, 75-87.

Press, W. C., S. A. Teukolsky, W. T. Vetterling, and B. P. Flannery, 1992: Numerical Recipes. Cambridge University Press, 963 pp.

Raupach, M. R., R. A. Antonia, and S. Rajagopalan, 1991: Roughwall turbulent boundary layers. Appl. Mech. Rev., 44, 1-25.

Sarghini, F., U. Piomelli, and E. Balaras, 1999: Scale-similar models for large-eddy simulations. Phys. Fluids, 11, 1596-1607.

Schumann, U., 1995: Stochastic backscatter of turbulence energy and scalar variance by random subgrid-scale fluxes. Proc. Roy. Soc. London, A451, 293-318.

Shaw, R. H., and U. Schumann, 1992: Large-eddy simulation of turbulent flow above and within a forest. Bound.-Layer Meteor., 61, 47-64.

__ J. Tavangar, and D. Ward, 1983: Structure of the Reynolds stress in a canopy layer. J. Climate Appl. Meteor., 22, 19221931.

_ _ K. T. Paw U, and W. Gao, 1989: Detection of temperature ramps and flow structures at a deciduous forest site. Agric. For. Meteor., 47, 123-138.

Sorbjan, Z., 1996: Effects caused by varying strength of the capping inversion based on a large-eddy simulation of the shear-free convective boundary layer. J. Atmos. Sci., 53, 2015-2024.

Su, H. H., R. H. Shaw, K. T. Paw U, C.-H. Moeng, and P. P. Sullivan, 1998: Turbulent statistics of neutrally stratified flow within and above a sparse forest from large-eddy simulation and field observations. Bound.-Layer Meteor., 88, 363-397.

Sullivan, P. P., J. C. McWilliams, and C.-H. Moeng, 1994: A subgridscale model for large-eddy simulation of planetary boundarylayer flows. Bound.-Layer Meteor., 71, 247-276.

Szilagyi, J., M. B. Parlange, G. G. Katul, and J. D. Albertson, 1999: An objective technique for determining principal time scales of 
coherent eddy structures using orthogonal wavelets. Adv. Water Res., 22, 561-566.

Tong, C., J. C. Wyngaard, S. Khanna, and J. G. Brasseur, 1998: Resolvable- and subgrid-scale measurement in the atmospheric surface layer: Technique and issues. J. Atmos. Sci., 55, 31143126.
,-- , and J. G. Brasseur, 1999: Experimental study of the subgrid-scale stresses in the atmospheric surface layer. J. Atmos. Sci., 56, 2277-2292.

Wyngaard, J. C., and S. F. Clifford, 1977: Taylor's hypothesis and high-frequency turbulence spectra. J. Atmos. Sci., 34, 922-929. 